\title{
On Unbounded Path-Loss Models: Effects of Singularity on Wireless Network Performance
}

\author{
Hazer Inaltekin, Mung Chiang, H. Vincent Poor, and Stephen B. Wicker
}

\begin{abstract}
This paper addresses the following question: how reliable is it to use the unbounded path-loss model $G(d)=d^{-\alpha}$, where $\alpha$ is the path-loss exponent, to model the decay of transmitted signal power in wireless networks? $G(d)$ is a good approximation for the path-loss in wireless communications for large values of $d$ but is not valid for small values of $d$ due to the singularity at 0 . This model is often used along with a random uniform node distribution, even though in a group of uniformly distributed nodes some may be arbitrarily close to one another. The unbounded path-loss model is compared to a more realistic bounded path-loss model, and it is shown that the effect of the singularity on the total network interference level is significant and cannot be disregarded when nodes are uniformly distributed. A phase transition phenomenon occurring in the interference behavior is analyzed in detail. Several performance metrics are also examined by using the computed interference distributions. In particular, the effects of the singularity at 0 on bit error rate, packet success probability and wireless channel capacity are analyzed.
\end{abstract}

Index Terms-Multiple-access interference, bounded path-loss models, unbounded path-loss models, bit error rate, packet success probability, wireless channel capacity.

\section{INTRODUCTION}

$\mathbf{F}$ OR THE purposes of mathematical tractability, it is necessary to have simple but useable abstractions of the wireless communication medium. An important example is the use of the function $G(d)=d^{-\alpha}$ to model the power gain of the wireless channel between a transmitter and a receiver, where $d$ is the distance between the two terminals and $\alpha$ is the path-loss exponent.

A key artifact of this model is the singularity at 0 . Even though the function is a reasonable approximation for wireless channel power gain for large transmitter-receiver separation, it becomes increasingly invalid as transmitters and receivers move closer to one another - i.e., the far-field assumption cannot be applied (see [1] for details on the far-field assumption). In particular, the singularity can lead to the physically impossible scenario in which the received signal power exceeds the transmitted signal power.

Manuscript received 27 August 2008; revised 31 January 2009. An earlier version of this work appeared in the Fourth Annual IEEE Communications Society Conference on Sensor, Mesh and Ad Hoc Communications and Networks, San Diego, CA, USA. This research was supported in part by the National Science Foundation under Grants ANI-03-58807, CNS-06-25637, CCF-0448012, and by ONR Grant N00014-07-1-0864.

Hazer Inaltekin, Mung Chiang, and H. Vincent Poor are with the Department of Electrical Engineering, Princeton University, Princeton, NJ, 08544 (e-mail: \{hinaltek, chiangm, poor\}@princeton.edu).

Stephen B. Wicker is with the School of Electrical and Computer Engineering, Cornell University, Ithaca, NY, 14850 (e-mail: wicker@ece.cornell.edu).

Digital Object Identifier 10.1109/JSAC.2009.090906.
In this paper, we examine the effects of the singularity at 0 in the unbounded path-loss model on network performance by comparing it with a bounded path-loss model when nodes are uniformly distributed over the network domain and use an Aloha type medium access control (MAC) protocol to gain the channel access. We focus on the large network limit where the number of nodes grows to infinity. We will show that a phase transition in the behavior of the network interference occurs at a critical value $\alpha^{*}$ of the path-loss exponent as the network grows to infinity. ${ }^{1}$ When $\alpha \leq \alpha^{*}$, the network multipleaccess interference behaves in a similar fashion under both bounded and unbounded path-loss models. When $\alpha>\alpha^{*}$, the network multiple-access interference behavior depends on path-loss models. In particular, the tails of the interference probability density function (PDF) are significantly affected by the singularity at 0 when $\alpha>\alpha^{*}$. The interference PDF becomes heavy-tailed under the unbounded path-loss model. On the other hand, it decays to zero exponentially under general bounded path-loss models with fading and random phase. These results hold independent of network user density, and are summarized in Table I.

We will use the results obtained for the interference distribution to compare bounded and unbounded path-loss models for three performance metrics: bit error rate (BER), packet success probability, and wireless channel capacity. We analyze these metrics as functions of the distance between a transmitterreceiver pair. Using the unbounded path-loss model results in significant deviations from more realistic performance figures obtained by using a bounded path-loss model for all three metrics. These results indicate that unbounded path-loss models should be used with caution in wireless communications and networking problems.

\section{A. Related Work}

In [2], the authors gave a comprehensive analysis on the behavior of one-dimensional shot noise by obtaining moments and moment generating functions for the shot-noise process. Interference modeling in wireless networks is a specific instance of a shot-noise process, and similar techniques are used to obtain the interference PDF for wireless networks in many previous studies such as [3], [4], [5] and [6]. In all these work, only the unbounded propagation model is considered. This is mainly due to the analytical simplicity of the calculations under this model.

\footnotetext{
${ }^{1}$ The expression "phase transition" is often used in the percolation theory and random graph literature while analyzing the existence of the unique giant connected component. In this paper, we use it to emphasize the dramatic change in the behavior of multiple-access network interference as a function of path-loss exponent.
} 
TABLE I

TABLE OF RESULTS

\begin{tabular}{|c|c|c|c|}
\hline Model & $\begin{array}{l}\text { Critical Path-loss } \\
\text { Exponent }\end{array}$ & $\begin{array}{c}\text { Interference Behavior } \\
\left(\alpha \leq \alpha^{*}\right)\end{array}$ & $\begin{array}{c}\text { Interference Behavior } \\
\left(\alpha>\alpha^{*}\right)\end{array}$ \\
\hline CSIM and BPM & $\alpha^{*}=4$ & $\begin{array}{c}\text { Diverges to Infinity } \\
\text { Rates: } r^{2-\frac{\alpha}{2}} \text { for } \alpha<\alpha^{*} \text { and } \log (r) \text { for } \alpha=\alpha^{*} \\
\text { (Theorem } 1)\end{array}$ & $\begin{array}{l}\text { Converges in Distribution } \\
\mathbb{P}\{|I|>x\}=o\left(e^{-x}\right) \\
\quad(\text { Theorems } 2 \text { and } 4)\end{array}$ \\
\hline CSIM and UPM & $\alpha^{*}=4$ & $\begin{array}{c}\text { Diverges to Infinity } \\
\text { Rates: } r^{2-\frac{\alpha}{2}} \text { for } \alpha<\alpha^{*} \text { and } \log (r) \text { for } \alpha=\alpha^{*} \\
(\text { Theorem } 1)\end{array}$ & $\begin{array}{l}\text { Converges in Distribution } \\
\mathbb{P}\{|I|>x\}=\Omega\left(x^{\frac{-4}{\alpha}}\right) \\
(\text { Theorems } 3 \text { and 5) }\end{array}$ \\
\hline USIM and BPM & $\alpha^{*}=2$ & $\begin{array}{c}\text { Diverges to Infinity } \\
\text { Rates: } r^{1-\frac{\alpha}{2}} \text { for } \alpha<\alpha^{*} \text { and } \sqrt{\log (r)} \text { for } \alpha=\alpha^{*} \\
\text { (Theorem } 6 \text { ) }\end{array}$ & $\begin{array}{c}\text { Converges in Distribution } \\
\mathbb{P}\{|I|>x\}=o\left(e^{-x}\right) \\
\text { (Theorems } 7 \text { and 9) }\end{array}$ \\
\hline USIM and UPM & $\alpha^{*}=2$ & $\begin{array}{c}\text { Diverges to Infinity } \\
\text { Rates: } r^{1-\frac{\alpha}{2}} \text { for } \alpha<\alpha^{*} \text { and } \sqrt{\log (r)} \text { for } \alpha=\alpha^{*} \\
\text { (Theorem 6) }\end{array}$ & $\begin{array}{c}\text { Converges in Distribution } \\
\mathbb{P}\{|I|>x\}=\Omega\left(x^{\frac{-4}{\alpha}}\right) \\
\text { (Theorems } 8 \text { and 10) }\end{array}$ \\
\hline
\end{tabular}

Table 1. CSIM and USIM stand for Correlated Signals Interference Model and Uncorrelated Signals Interference Model, respectively. BPM and UPM stand for Bounded Path-loss Model and Unbounded Path-loss Model, respectively. $\alpha$ is the path-loss exponent. $r$ is the radius of the network domain. $I$ is the network multiple-access interference at the large system limit. See Section II for details.

More recent studies along this line of research are [7], [8], [9], [10] and [11]. In [7], the authors considered the signal-tointerference ratio for wireless ad hoc networks with randomly distributed nodes, and obtained bounds on its distribution. In [8], the authors analyzed the sizes and shapes of cells in a wireless cellular code-division multiple-access (CDMA) network by using marked Poisson processes and the shotnoise process associated with the marked point process under various path-loss models. The papers [9] and [10] analyzed the effect of unbounded path-loss models on the transport capacity of wireless networks. In [11], the authors considered the interference and outage probability in clustered wireless ad hoc networks for bounded and unbounded path-loss models. Our results are closest to these latter results in that they also obtained similar power-law decaying and exponential decaying interference distribution results under their path-loss models. However, our physical layer model and interference models are different than those of [11]. In this paper, we obtain interference signal strength PDFs and other network parameters under both bounded and unbounded propagation models, and analyze the effect of the singularity at 0 by comparing them.

\section{Problem Formulation}

\section{A. Notation}

As in standard notation, for any two real-valued functions $f$ and $g$, we write $f(x)=O(g(x))$ as $x \rightarrow \infty$ if $\limsup _{x \rightarrow \infty}\left|\frac{f(x)}{g(x)}\right|<\infty$. We write $f(x)=o(g(x))$ as $x \rightarrow \infty$ if $\lim _{x \rightarrow \infty} \frac{f(x)}{g(x)}=0$. We write $f(x)=\Omega(g(x))$ as $x \rightarrow \infty$ if $\liminf _{x \rightarrow \infty}\left|\frac{f(x)}{g(x)}\right|>0$. We write $f(x) \sim g(x)$ as $x \rightarrow \infty$ if $\lim _{x \rightarrow \infty} \frac{f(x)}{g(x)}=1$.

We represent the set of complex numbers and the set of real numbers by $\mathbb{C}$ and $\mathbb{R}$, respectively. For a random variable $X$, we define the Laplace transform of its distribution as $\varphi_{X}(s)=\mathbb{E}\left[e^{s \cdot X}\right]$, where $s=\theta+\dot{u} t \in \mathbb{C}, \theta \in \mathbb{R}, t \in \mathbb{R}$ and $\dot{u}^{2}=-1$. We use $\Re(s)=\theta$ to represent the real part of $s$. When $\varphi_{X}(s)$ is calculated on the imaginary line, i.e., $s=\dot{a} t$, we obtain the characteristic function of $X$. With a slight abuse of notation, we also represent its characteristic function as $\varphi_{X}(t)=\mathbb{E}\left[e^{\dot{a t} \cdot X}\right]$. Note that characteristic functions of

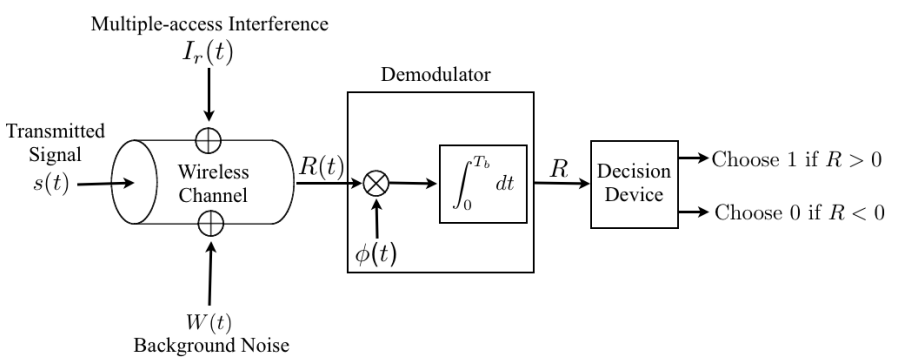

Fig. 1. Physical layer model for decoding of transmitted bits.

random variables are always defined, and we use them to obtain the network interference probability density function in the large system limit. For calculating the decay rate of the network interference PDF, Laplace transforms will be helpful.

For a sequence of random variables $\left\{X_{n}\right\}_{n=1}^{\infty}, X_{n} \stackrel{i . p .}{\rightarrow} X$ means that the sequence converges to $X$ in probability. $X_{n} \Rightarrow$ $X$ means that it converges to $X$ in distribution. We say a random variable $X$ has a heavy-tailed distribution if $\mathbb{P}\{|X|>$ $x\}=\Omega\left(x^{-\theta}\right)$ for some $\theta>0 . \mathcal{N}\left(0, \sigma^{2}\right)$ denotes a Gaussian random variable with zero mean and variance $\sigma^{2}$.

\section{B. Physical Layer and Network Model}

We consider a disk shaped network domain $\mathcal{B}(\mathbf{0}, r)$ centered at the origin $\mathbf{0}$ and having radius $r \in \mathbb{R}$. Transmitters are uniformly distributed over the network domain.

For the ease of mathematical exposition, network nodes are assumed to use the binary phase shift keying (BPSK) modulation scheme for information transmission without any power control algorithm at the physical layer. Each transmitter transmits with a fixed power $P=\frac{E_{b}}{T_{b}}$, where $E_{b}$ is the transmitted signal energy per bit, and $T_{b}$ is the bit duration. The pair of signals $s_{0}(t)=-\sqrt{\frac{2 E_{b}}{T_{b}}} \cos \left(2 \pi f_{c} t\right)$ and $s_{1}(t)=$ $\sqrt{\frac{2 E_{b}}{T_{b}}} \cos \left(2 \pi f_{c} t\right)$, for $0 \leq t \leq T_{b}$, are used to transmit binary symbols 0 and 1 , respectively. At the receiver side, transmitted signals are coherently decoded by using the basis function $\phi(t)=\sqrt{\frac{2}{T_{b}}} \cos \left(2 \pi f_{c} t\right), 0 \leq t \leq T_{b}$. The physical layer model for decoding transmitted bits is depicted in Fig. 1.

Transmitted signals are impaired by the interference coming from other transmitters and the white noise process $W(t)$ as 
shown in Fig. 1. Therefore, the received signal $R$ at the demodulator output in Fig. 1 contains the desired signal coming from the transmitted signal to be decoded, the noise signal $W_{0}$ coming from $W(t)$, and the multiple-access interference signal $I_{r}$ coming from the transmitters transmitting concurrently. We place a test receiver node at the origin, and focus on $I_{r}$ at the test receiver node. We will study the convergence properties of $I_{r}$ at the test receiver node as $r$ grows to infinity. We represent the limiting network multiple-access interference by $I$ as $r$ grows to infinity. Let $\lambda>0$ be the density of transmitters, which is in units of number of nodes per unit area. We pick a transmitter density $\lambda>0$, and keep it constant while growing the network size to infinity. The total number of interfering transmitters for any given value of the network radius $r$ is given by $\left\lceil\lambda \pi r^{2}\right\rceil$, where $\lceil u\rceil$ denotes the smallest integer larger than $u \in \mathbb{R}$.

\section{Path-loss Models}

We classify path-loss models as being either bounded or unbounded. For the unbounded path-loss model (UPM), we focus on the signal strength decay function $G_{1}(d)=\frac{1}{d^{\frac{\alpha}{2}}}$. Therefore, the transmitted signal power decays according to the commonly used signal power attenuation function $G(d)=$ $\frac{1}{d^{\alpha}}$. This model is not correct for small values of $d$ due to a singularity at 0 . However, it fairly well approximates the signal attenuation for large values of $d$.

In contrast to the unbounded path-loss model, a sound propagation model for characterizing signal strength attenuation must always be a monotonically decreasing function of the distance and be bounded by unity. In addition, received signal power under this model must behave asymptotically as $\frac{1}{d^{\alpha}}$ as $d \rightarrow \infty$. A useful such model for the received signal strength is $G_{2}(d)=\frac{1}{1+d^{\frac{\alpha}{2}}}$, which we will consider as our bounded path-loss model (BPM). It is a reasonable way of improving the inverse-power law model by removing the singularity at 0 . Note that $G_{2}(0)=1$, which means that when a transmitter and a receiver are co-located, the receiver receives exactly what the transmitter transmits. Our results on the decay rate of the network interference PDF will be proven for general bounded path-loss functions.

\section{Interference Models}

Our interference models are similar to the models presented in [4], [13] and [14]. As in these work, we assume that the total network multiple-access interference is equal to the summation of demodulated residual signals coming from all interfering transmitters in the network. Our characterizations of the decay rate of the network interference PDF for bounded path-loss functions are developed by considering random phases and fading coefficients. However, we assume perfect phase synchronization and no fading while comparing network performance metrics under the BPM and the UPM. For notational simplicity, we present our interference models without random phases and fading coefficients.

We consider two interference models. In the first model, all interferers transmit the same symbols, and therefore interference signals are perfectly correlated. This model is appropriate for multiple-access interference calculations in sensor networks since sensor readings tend to be correlated with each other. Under this model, which we call Correlated Signals Interference Model (CSIM), $I_{r}$ can be written as $I_{r}=\sum_{k=1}^{\left\lceil\lambda \pi r^{2}\right\rceil} I_{k}^{(r)}$, where $I_{k}^{(r)}$ is the interference coming from the $k^{\text {th }}$ transmitter at the demodulator output of the receiver when the network radius is equal to $r . I_{k}^{(r)}$ is equal to $I_{k}^{(r)}=Z \frac{\sqrt{E_{b}}}{1+\left\|X_{k}^{(r)}\right\|^{\frac{\alpha}{2}}} \quad$ under the BPM, and $I_{k}^{(r)}=$ $Z \frac{\sqrt{E_{b}}}{\left\|X_{k}^{(r)}\right\|^{\frac{\alpha}{2}}}$ under the UPM, where $Z$ is a random variable taking values \pm 1 with equal probabilities of $\frac{1}{2}$, and $X_{k}^{(r)}$ is a random variable representing the location of the $k^{\text {th }}$ transmitter, which is uniformly distributed over $\mathcal{B}(\mathbf{0}, r) . Z=1$ $(Z=-1)$ means that all interferers transmit the binary symbol 1 (0). When we write $\left\|X_{k}^{(r)}\right\|$, we mean the distance of the $k^{\text {th }}$ transmitter to the origin. $Z$ and $X_{k}^{(r)}$,s are independent from one another.

CSIM contains best-case and worst-case scenarios. In the best-case, the symbol to be decoded at the test receiver becomes equal to the symbol transmitted by other transmitters. The signal quality at the test receiver is enhanced by other transmitters. In the worst-case, the symbol to be decoded at the test receiver becomes different than the symbol transmitted by other transmitters. In this case, the signal quality is deteriorated by other transmitters. The average network performance under CSIM becomes equal to the statistical average of worstcase and best-case scenarios.

Our second interference model is proposed to capture the possibility that different transmitters in a wireless network may transmit different symbols. Under the second model, which we call Uncorrelated Signals Interference Model (USIM), individual interference signals at the demodulator output of the test receiver are given as $I_{k}^{(r)}=Z_{k} \frac{\sqrt{E}_{b}}{1+\left\|X_{k}^{(r)}\right\|^{\frac{\alpha}{2}}}$ under the BPM, and $I_{k}^{(r)}=Z_{k} \frac{\sqrt{E_{b}}}{\left\|X_{k}^{(r)}\right\|^{\frac{\alpha}{2}}}$ under the UPM, where the $Z_{k}$ 's are independent random variables taking values \pm 1 with equal probabilities of $\frac{1}{2}$. We assume that the $Z_{k}$ 's are also independent of $X_{k}^{(r)}$. The total interference signal at the demodulator output of the test receiver is equal to the summation of $I_{k}^{(r)}$ 's.

\section{INTERFERENCE DISTRIBUTION - CORRELATED SignAls INTERFERENCE MODEL}

In this section, we present interference PDF calculations under different path-loss models for CSIM. We will look at the asymptotic distribution of $I_{r}$ as $r \rightarrow \infty$. As a result of our analysis, we will conclude that a phase transition occurs at the critical value of $\alpha=4$, below which network interference behaves the same under both the UPM and BPM, and above which it has very different characteristics under the two pathloss models. We start with the case $\alpha \leq 4$.

\section{A. Interference Behavior for $\alpha \leq 4$ :}

We first look at the interference asymptote as network size grows to infinity when $\alpha \leq 4$. In particular, we will show that interference goes to infinity in probability and the rate at which it goes to infinity is $r^{2-\frac{\alpha}{2}}$ for $\alpha<4$ and $\log (r)$ for $\alpha=4$. The following theorem is the central result of this subsection. A similar result for marked Poisson processes first 
appeared in [15]. Here, we extend the results in [15] to the case where locations of nodes are uniformly distributed as well as providing rates of convergence of $I_{r}$ to infinity.

Theorem 1: Under both the UPM and BPM, if $\alpha<4$, $\frac{I_{r}}{r^{2-\frac{\alpha}{2}}} \stackrel{i . p .}{\rightarrow} Z \frac{4 \lambda \pi}{4-\alpha} \sqrt{E_{b}}$, and if $\alpha=4, \frac{I_{r}}{\log (r)} \stackrel{i . p .}{\rightarrow} 2 Z \sqrt{E_{b}} \lambda \pi$.

Proof: See Appendix A.

A key conclusion from Theorem 1 is that the interference signal strength does not converge in distribution to a real valued random variable for either of the path-loss models when $\alpha \leq 4$. In fact, the interference magnitude goes to infinity in probability at the same rate under both models. The intuitive explanation for such behavior is that the interference is determined by the number of interferers rather than the properties of the propagation models when $\alpha \leq 4$. That is, if $\alpha \leq 4$, interference signal strength from any individual interferer decays at a rate smaller than $O\left(r^{-2}\right)$. However, in order to keep the density of the transmitters constant, we increase their number at rate $O\left(r^{2}\right)$. Since the decay rate of the propagation models is not fast enough, addition of the small but relatively large number of interference signals results in the convergence of the interference magnitude to infinity.

The results of III-B in conjunction with Theorem 1 will prove the existence of phase transition phenomena in the interference behavior at $\alpha=4$. In particular, it will be shown that whenever $\alpha>4$, interference signal strength converges in distribution as the network size grows to infinity, and the limiting interference distribution behaves very differently under our two path-loss models.

\section{B. Interference Behavior for $\alpha>4$ :}

We will analyze the asymptotic distribution of the interference for the case $\alpha>4$. The technique is classical in that we will first obtain the characteristic function of the interference, and then invert it to obtain the interference $\mathrm{PDF}^{2}$ We give the derivation for the BPM in Appendix B. The derivation for the UPM is similar.

Theorem 2: The interference characteristic function under the BPM and CSIM is given by

$\lim _{r \rightarrow \infty} \varphi_{I_{r}}(t)=\varphi_{I}(t)=\Re\left(\exp \left(\frac{-4 \lambda \pi}{\alpha} E_{b}^{\frac{2}{\alpha}} t^{\frac{4}{\alpha}} C(t)\right)\right)$,

where $C(t)=\int_{0}^{t \sqrt{E_{b}}}(1-\exp (\dot{u} u)) \frac{\left(1-\frac{u}{t \sqrt{E_{b}}}\right)^{\frac{4}{\alpha}-1}}{u^{\frac{4}{\alpha}+1}} d u$.

Proof: See Appendix B

Theorem 3: The interference characteristic function under the UPM and CSIM is given by

$$
\begin{aligned}
& \lim _{r \rightarrow \infty} \varphi_{I_{r}}(t)=\varphi_{I}(t) \\
& =\Re\left(\exp \left(\frac{-4 \lambda \pi}{\alpha} E_{b}^{\frac{2}{\alpha}}\left(\mathbb{1}_{\{t \geq 0\}} C+\mathbb{1}_{\{t<0\}} C^{*}\right)|t|^{\frac{4}{\alpha}}\right)\right)
\end{aligned}
$$

where $C=\int_{0}^{\infty}(1-\exp (\dot{u} u)) u^{\frac{-4}{\alpha}-1} d u, C^{*}$ is the complex conjugate of $C$.

We now describe some simulation studies that illustrate and verify the above analytical results. We invert the characteristic functions given above numerically to obtain the interference [16]).
PDF. We also perform Monte Carlo simulations to obtain the histogram of the network interference. As seen in Figs. 2 and 3 , there is a very close match between the interference PDFs obtained in these two ways.

The interference PDF under the BPM in Fig. 2 decays exponentially, whereas its decay rate under the UPM in Fig. 3 is much slower. Decay rates of interference distributions are given in Theorems 4 and 5. Theorem 4 characterizes the decay rate of the interference distribution under general bounded path-loss models, fading and random phase. We do not provide the proof for Theorem 4 since a similar proof will be given for USIM.

Theorem 4: Let $\mathbb{P}\{|I|>x\}$ be the probability that the interference magnitude is greater than $x>0$ under a general bounded path-loss function $G_{B}$ and the CSIM. Let $G_{B}$ : $[0, \infty) \mapsto(0, \infty)$ satisfy the following properties. ${ }^{3}$

- Smoothness: $G_{B}(d)$ is almost everywhere differentiable, and a non-increasing function of the distance $d$. Moreover, there exists a $T>0$ such that its functional inverse $G_{B}^{-1}$ is well-defined for all $d \geq T$.

- Boundedness: $G_{B}(0)<\infty$.

- Decay Rate: $G_{B}(d) \sim d^{-\frac{\alpha}{2}}$ as $d \rightarrow \infty$.

It is assumed that the phase of each interference signal is shifted by the same amount according to a phase distribution that is symmetric over $[-\pi, \pi]$. It is also assumed that the magnitude of each interference signal is independently scaled by a fading coefficient whose Laplace transform is welldefined for all $\Re(s)>0 .{ }^{4}$ Then, $\mathbb{P}\{|I|>x\}=o\left(e^{-x}\right)$ as $x \rightarrow \infty$. In particular,

$$
\lim _{x \rightarrow \infty}\left|\frac{\log (\mathbb{P}\{|I|>x\})}{x}\right|=\infty .
$$

Theorem 5: Let $\mathbb{P}\{|I|>x\}$ be the probability that the interference magnitude is greater than $x>0$ under the UPM and CSIM. Then, $\mathbb{P}\{|I|>x\}=\Omega\left(x^{\frac{-4}{\alpha}}\right)$ as $x \rightarrow \infty$.

Proof: See Appendix C.

\section{INTERFERENCE DISTRIBUTION - UNCORRELATED SignAls INTERFERENCE MODEL}

Our aim now is to extend the analysis presented in the previous section to the second interference model (USIM). Recall that interference signals are added constructively and destructively with equal probability under USIM. A similar phase transition phenomenon also occurs under USIM. This time, the critical value for $\alpha$ is 2 . We start with the case $\alpha \leq 2$.

\section{A. Interference Behavior for $\alpha \leq 2$ :}

The main result of this subsection is that if the multipleaccess network interference is scaled with $r^{1-\frac{\alpha}{2}}$ for $\alpha<2$ and with $\sqrt{\log (r)}$ for $\alpha=2$, it converges in distribution to a Gaussian random variable.

\footnotetext{
${ }^{3}$ Some examples for bounded path-loss functions satisfying these conditions are $\max \left(A, d^{\frac{-\alpha}{2}}\right), \frac{1}{(1+d)^{\frac{\alpha}{2}}}$ and $\frac{1}{1+d^{\frac{\alpha}{2}}}$.

${ }^{4}$ The widely used Rayleigh, Rician and Nakagami fading models satisfy this condition.
} 


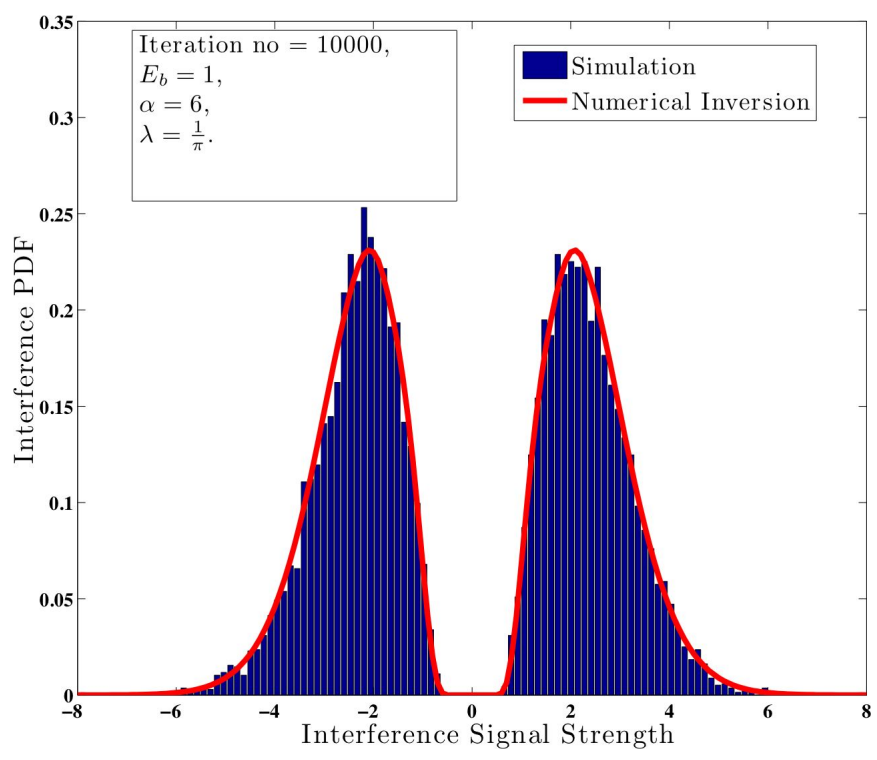

Fig. 2. Interference PDF under the BPM from simulation and from numerical inversion of the characteristic function for CSIM.

Theorem 6: Under both the UPM and BPM, if $\alpha<$ $2, \frac{I_{r}}{r^{1-\frac{\alpha}{2}}} \Rightarrow \mathcal{N}\left(0, \frac{2 \lambda \pi E_{b}}{2-\alpha}\right)$, and if $\alpha=2, \frac{I_{r}}{\sqrt{\log (r)}} \Rightarrow$ $\mathcal{N}\left(0,2 E_{b} \lambda \pi\right)$.

Proof: See Appendix D.

\section{B. Interference Behavior for $\alpha>2$ :}

We now provide network interference characteristic functions under USIM in Theorems 7 and 8 for both BPM and UPM. The derivations of these interference characteristic functions are similar to the derivation given in Appendix B under CSIM for BPM. Therefore, we do not give their derivations due to space limitations, and refer interested users to [18]. Similar results for the UPM also appeared in [4] and [6].

Theorem 7: The interference characteristic function under the BPM and USIM is given by

$$
\lim _{r \rightarrow \infty} \varphi_{I_{r}}(t)=\varphi_{I}(t)=\exp \left(-\frac{4 \lambda \pi}{\alpha} E_{b}^{\frac{2}{\alpha}} C(|t|)|t|^{\frac{4}{\alpha}}\right),
$$

where $C(t)=\int_{0}^{t \sqrt{E_{b}}} \frac{(1-\cos (u))}{u^{\frac{4}{\alpha}+1}}\left(1-\frac{u}{t \sqrt{E_{b}}}\right)^{\frac{4}{\alpha}-1} d u$.

Theorem 8: The interference characteristic function under the UPM and USIM is given by

$$
\lim _{r \rightarrow \infty} \varphi_{I_{r}}(t)=\varphi_{I}(t)=\exp \left(-\frac{4 \lambda \pi}{\alpha} E_{b}^{\frac{2}{\alpha}} C|t|^{\frac{4}{\alpha}}\right),
$$

where $C=\int_{0}^{\infty}(1-\cos (u)) u^{\frac{-4}{\alpha}-1} d u$.

An important distinction between the characteristics of the interference signal strength under CSIM and USIM is that as the network size grows to infinity, it converges in distribution for the values of $\alpha \in(2,4]$ under USIM, whereas it diverges to infinity under CSIM for any $\alpha \in(2,4]$. For example, if $\alpha=4$, the interference PDF becomes a Cauchy distribution with median zero under UPM for USIM. Therefore, we conclude that positive and negative additions of interference signals contribute additional stability to the total amount of network interference.

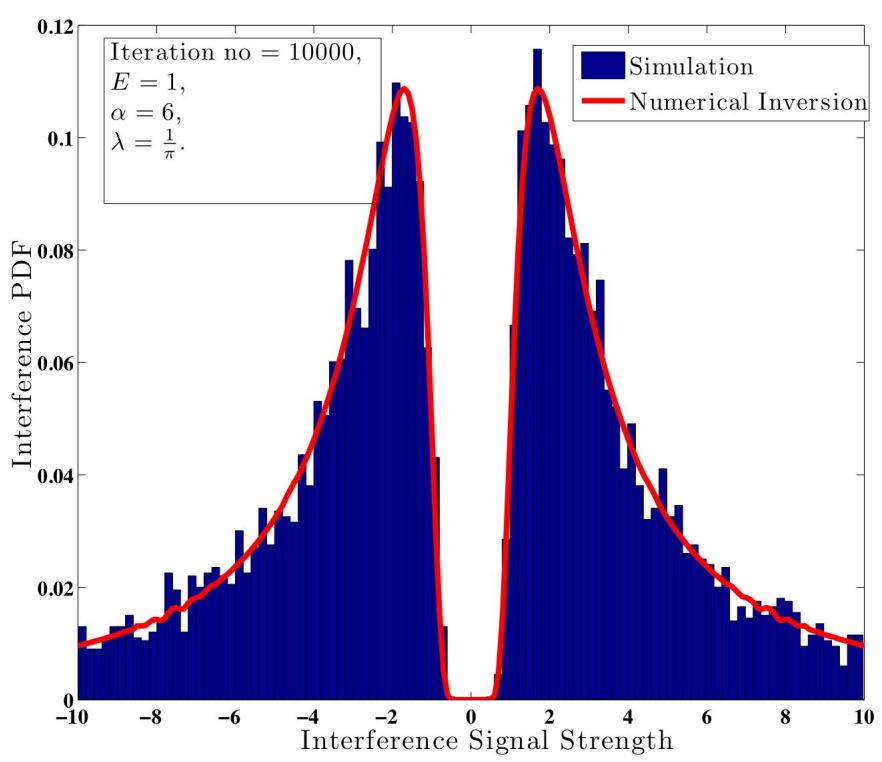

Fig. 3. Interference PDF under the UPM from simulation and from numerical inversion of the characteristic function for CSIM.

In Figs. 4 and 5, we show interference PDFs obtained after the numerical inversion of the characteristic functions in Theorems 7 and 8, and from Monte Carlo simulation. Again, we observe a very close match of simulation results and the numerical inversion. This verifies our calculations.

As we see from these figures, the interference PDF appears to decay exponentially under BPM. On the other hand, the decay rate of the interference is very slow under UPM. Decay rates of multiple-access network interference distributions are given in Theorems 9 and 10. Theorem 9 is again given for general bounded path-loss models under fading and random phase.

Theorem 9: Let $\mathbb{P}\{|I|>x\}$ be the probability that the interference magnitude is greater than $x>0$ under a general bounded path-loss function $G_{B}$ and the USIM. Let $G_{B}$ : $[0, \infty) \mapsto(0, \infty)$ satisfy the following properties.

- Smoothness: $G_{B}(d)$ is almost everywhere differentiable, and a non-increasing function of the distance $d$. Moreover, there exists a $T>0$ such that its functional inverse $G_{B}^{-1}$ is well-defined for all $d \geq T$.

- Boundedness: $G_{B}(0)<\infty$.

- Decay Rate: $G_{B}(d) \sim d^{-\frac{\alpha}{2}}$ as $d \rightarrow \infty$.

It is assumed that the phase of each interference signal is independently shifted according to the same phase distribution that is symmetric over $[-\pi, \pi]$. It is also assumed that the magnitude of each interference signal is independently scaled by a fading coefficient whose Laplace transform is welldefined for all $\Re(s)>0$. Then, $\mathbb{P}\{|I|>x\}=o\left(e^{-x}\right)$ as $x \rightarrow \infty$. In particular,

$$
\lim _{x \rightarrow \infty}\left|\frac{\log (\mathbb{P}\{|I|>x\})}{x}\right|=\infty .
$$

Proof: See Appendix E.

Theorem 10: Let $\mathbb{P}\{|I|>x\}$ be the probability that the interference magnitude is greater than $x>0$ under UPM and USIM. Then, $\mathbb{P}\{|I|>x\}=\Omega\left(x^{\frac{-4}{\alpha}}\right)$ as $x \rightarrow \infty$.

Proof: See Appendix F. 


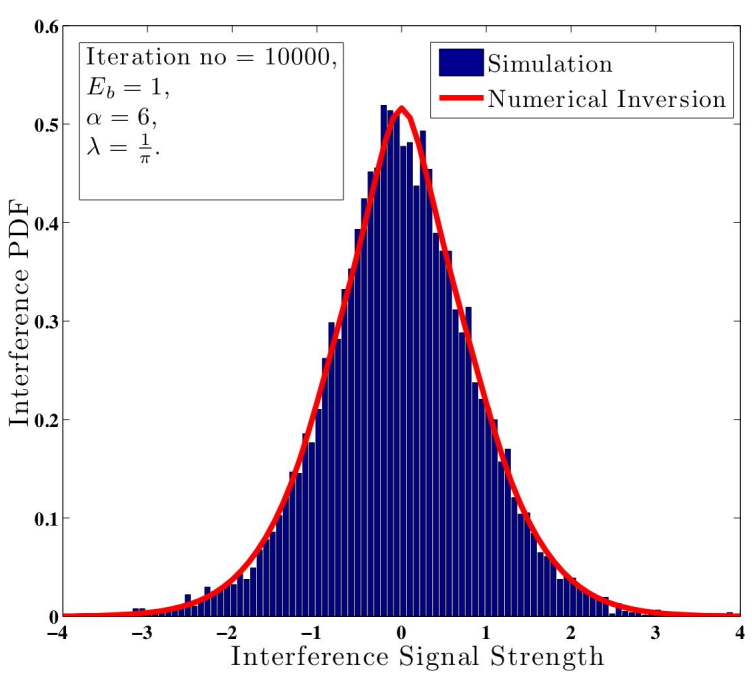

Fig. 4. Interference PDF under the BPM from simulation and from numerical inversion of the characteristic function for USIM.

\section{Effect on Bit Error Rate, Packet Success} Probability AND Wireless ChanNEL CAPACITY

In this section, we will analyze the effect of the singularity at 0 in the UPM on various performance metrics such as steady state BER, packet success probability and wireless channel capacity. Our aim is to understand the consequences of using the unbounded path-loss model on concrete metrics by using the PDFs obtained in the previous sections.

\section{A. Steady State Bit Error Rate}

We will first analyze the steady state BER under bounded and unbounded path-loss models. BER is an important performance metric which in turn helps us to determine packet success probability and wireless channel capacity.

We assume that a transmitted information bit is successfully decoded if and only if the interference plus noise level at the demodulator output of the receiver is sufficiently small enough. Let $b$ be a generic transmitted bit, and $\mathcal{E}(d)$ be the event that $b$ is decoded erroneously at the receiver when transmitter-receiver separation is equal to $d$. Due to the symmetry of the problem, we have $\mathbb{P}\{\mathcal{E}(d)\}=\frac{1}{2} \mathbb{P}\{\mathcal{E}(d) \mid b=$ $1\}+\frac{1}{2} \mathbb{P}\{\mathcal{E}(d) \mid b=0\}=\mathbb{P}\{\mathcal{E}(d) \mid b=1\}$.

Recall that transmitted bits are also impaired by the white noise process $W(t)$ with power spectral density $\frac{N_{0}}{2}$. Let $W_{0}$ be the corresponding noise at the demodulator output of the receiver coming from $W(t)$. Then, $W_{0}$ is a Gaussian random variable with mean zero and variance $\frac{N_{0}}{2}$. We also assume that interference signal reduction by $A$ times is possible at receiver nodes. $A=1$ corresponds to the classical narrowband digital communication, and $A>1$ can be thought of as corresponding to a broadband communication scheme such as CDMA ([20]). As a result,

$\mathbb{P}\{\mathcal{E}(d)\}=\frac{1}{2} \int_{-\infty}^{\infty} f_{I}(x) \operatorname{erfc}\left(\sqrt{\frac{E_{b} G_{i}(d)^{2}}{N_{0}}}+\frac{x}{A \sqrt{N_{0}}}\right) d x$,

where $f_{I}(x)$ is the probability density function of the multipleaccess interference signal $I$ coming from other terminals transmitting concurrently and $G_{i}, i=1,2$, is the path-loss

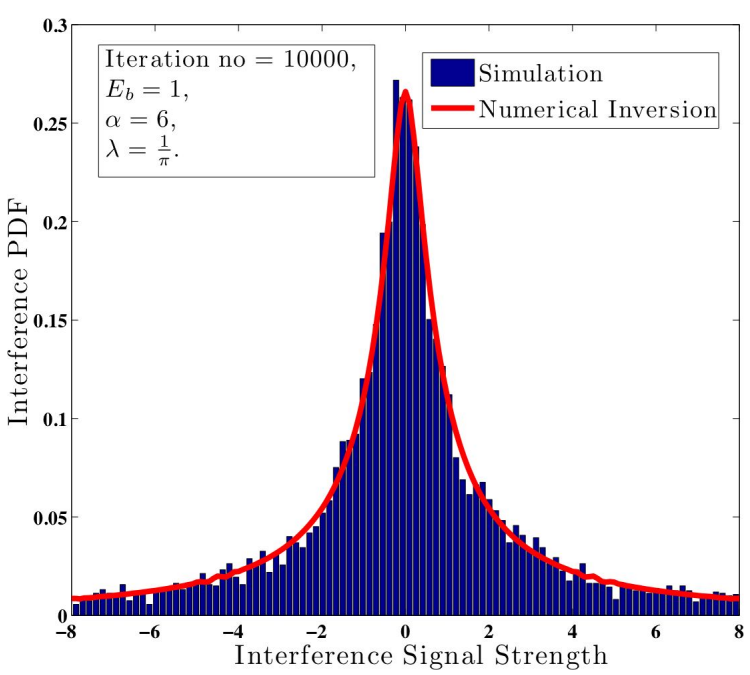

Fig. 5. Interference PDF under the UPM from simulation and from numerical inversion of the characteristic function for USIM.

function. Below, we plot the steady state BER as a function of transmitter and receiver separation $d$ for both high-noise regime and low-noise regime. For the high-noise regime, we assume that the background noise power is comparable with the transmitted energy per bit. To this end, we set $N_{0}=0.5$ and $E_{b}=1$. For the low-noise regime, we assume that the background noise power is much smaller than the transmitted energy per bit. To this end, we set $N_{0}=0.01$ and $E_{b}=1$ for the low-noise regime. The shape of the BER curves as functions of $d$ depends on the specific selection of the parameter $A$, the interference model and the path-loss model. Below, we describe results for two different values of $A$ under different path-loss and interference models. It is also assumed that $\alpha=6$ and $\lambda=\frac{1}{\pi}$.

Examining Fig. 6, we observe that BER approaches 0 when $d$ goes to zero under the UPM. In fact, this is the typical behavior of the BER under the UPM for any value of $A$. The main reason for such behavior is the unrealistic singularity of the UPM at 0 . Received signal energy per bit increases unboundedly as the transmitter-receiver separation is made smaller and smaller, which results in the convergence of the BER to 0 no matter how big the interference plus noise signal strength is. Especially for dense wireless networks where transmitters and receivers become arbitrarily close to one another with high probability, this has far more dramatic effects on the data transmission rate, which further leads to very unrealistic estimates for the number of successfully communicating transmitter-receiver pairs.

If we look at Fig. 6 again, we observe that the BER is bounded away from 0 under the BPM since the received signal energy per bit can be at most 1 [unit energy]. Therefore, whenever the interference plus noise level is high enough at the demodulator output of the receiver, the detector decodes transmitted bits erroneously.

Similar qualitative conclusions continue to hold in other figures as well. When the UPM is used to model the physical layer of a wireless network, the BER is underestimated for small values of transmitter-receiver separation. This is due to received power being unboundedly large when transmitters 


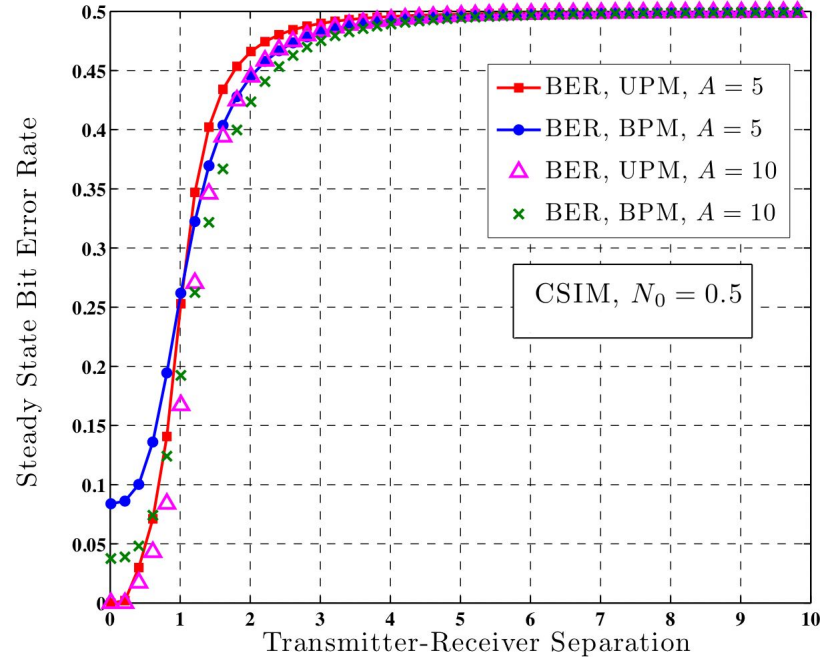

Fig. 6. Steady state BER as a function of transmitter-receiver separation under CSIM for the high-noise regime.

and receivers are arbitrarily close to one another. For moderate to high values of transmitter-receiver separation, we start to overestimate the BER under the UPM. This is because interference signal strength PDFs are more spread to the left and right under the UPM which results in theoretically higher interference signal levels at receiver nodes.

For the low-noise regime where transmitted bits are mainly impaired by multiple access interference coming from other transmitters, it is also possible to obtain BERs close to 0 for small values of transmitter-receiver separation under the BPM but for completely other reasons. For small values of transmitter-receiver separation, a bit is decoded in error if and only if the multiple access interference is high enough when the background noise is so small compared with the transmitted energy per bit. However, the tails of interference PDFs decay exponentially fast under the BPM, and the area underneath of these tails becomes negligible after high enough interference values. Therefore, the BER becomes close to zero under the BPM when transmitter-receiver separation is small enough in the low-noise regime (see Fig. 7 and Fig. 9).

\section{B. Packet Success Probability}

Next, we would like to compare packet success probability as a function of transmitter-receiver separation under different path-loss models. We assume that digital data is encoded by means of an error control coding technique (see [21]) into packets of $D \geq 1$ bits, and a packet fails if and only if there are more than $L, 1 \leq L \leq D$, bit errors inside the packet. For the purposes of calculating packet success probability, we consider two extreme regimes of node mobility. The first one is the fast-mobility regime where transmitter locations shuffle sufficiently enough during the transmission of a bit that bit errors can be assumed to be independent. The second regime is the slow-mobility regime where nodes can be assumed to be static over the course of a packet transmission. Note that bit errors are dependent in the slow-mobility regime since locations of transmitters do not change dramatically during a bit duration.

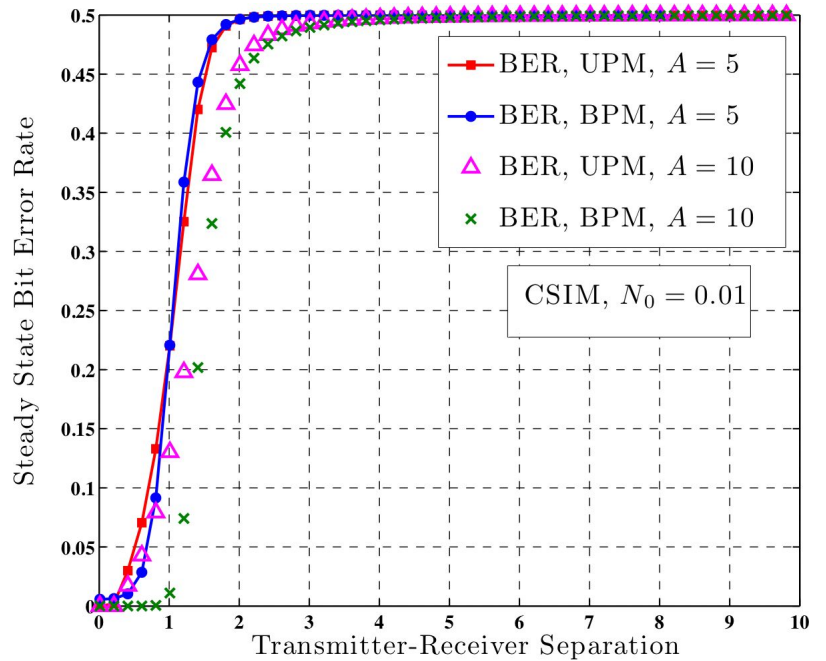

Fig. 7. Steady state BER as a function of transmitter-receiver separation under CSIM for the low-noise regime.

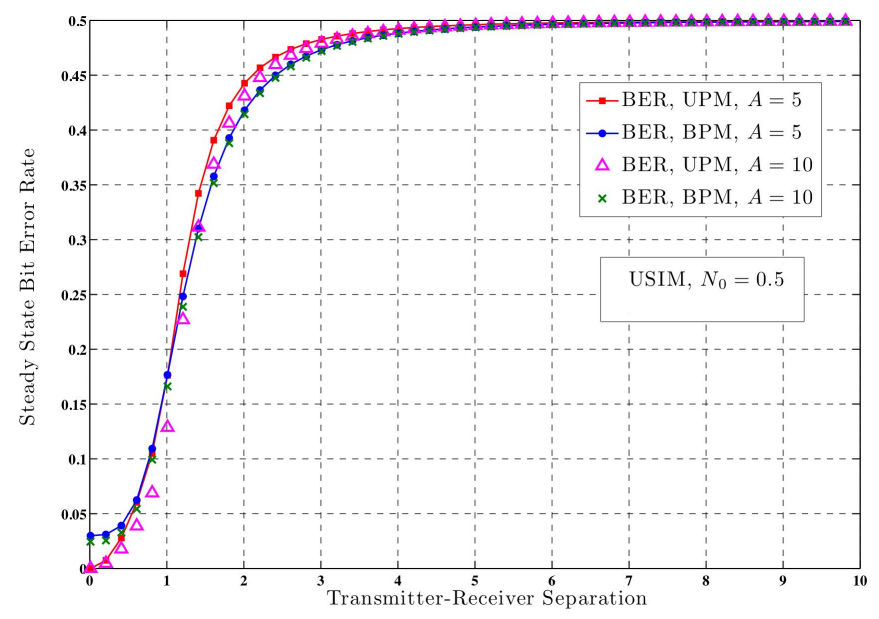

Fig. 8. Steady state BER as a function of transmitter-receiver separation under USIM for the high-noise regime.

We start with calculating packet success probability for the fast-mobility regime. For simplicity, we assume that the separation between our transmitter and receiver reference nodes is fixed at $d$. If it is random, one needs to average packet success probability one more time by using the distribution of $d$. Let $\mathrm{BER}_{i}(d), i=1,2$, denote the steady state bit error rates calculated in V-A under different interference models when transmitter-receiver separation is equal to $d . i=1$ corresponds to the UPM, and $i=2$ corresponds to the BPM. Let $\mathcal{S}(d)$ be the event that a transmitted packet is decoded successfully at the receiver when the transmitter-receiver separation is equal to $d$. Then, for different path-loss models, the packet success probability in the fast-mobility regime is equal to $\mathbb{P}(\mathcal{S}(d))=\sum_{e=0}^{L}\left(\begin{array}{l}D \\ e\end{array}\right) \operatorname{BER}_{i}(d)^{e}\left(1-\operatorname{BER}_{i}(d)\right)^{D-e}$.

The change of packet success probability as a function of transmitter-receiver separation in the fast-mobility regime is depicted in Fig. 10 for $E_{b}=1, \alpha=6, \lambda=\frac{1}{\pi}$ and $A=5$. The results shown in Fig. 10 are for $D=100$ and $L=10$. To cover as many different cases as possible, we consider the highnoise regime for CSIM and the low-noise regime for USIM. As we observe in Fig. 10, there is a significant difference 


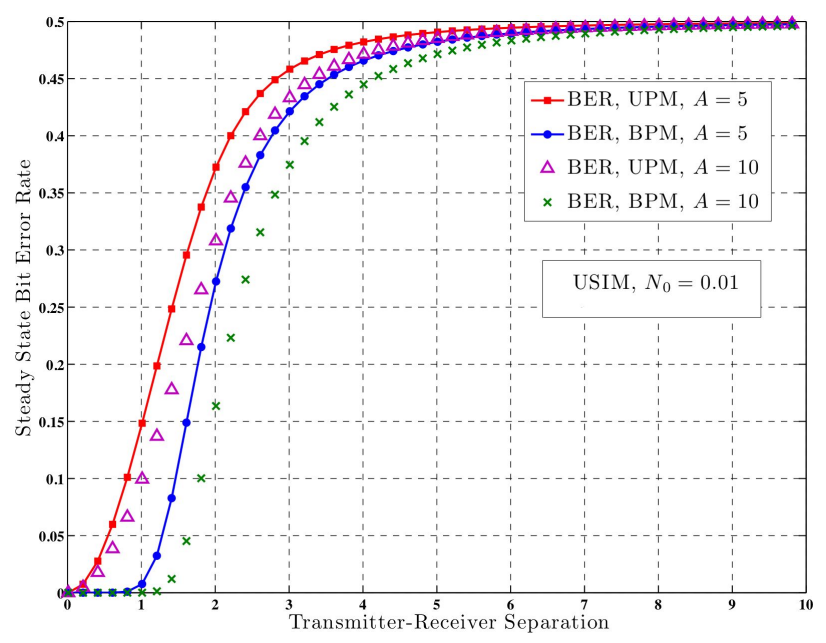

Fig. 9. Steady state BER as a function of transmitter-receiver separation under USIM for the low-noise regime.

between packet success probability curves under different path-loss models. The UPM can overestimate or underestimate the packet success probability substantially depending on the specific selection of interference models and other network parameters. The packet success probability becomes close to 1 for small values of transmitter-receiver separation under both path-loss models as the received signal strength becomes more dominant than the total network multiple-access interference plus noise while decoding transmitted bits. For large values of transmitter-receiver separation, the packet success probability becomes close to 0 under both path-loss models since the received signal strength becomes negligibly small when compared with the total network multiple-access interference plus noise. For values of transmitter-receiver separation close to 0 , the UPM overestimates the packet success probability since the received signal strength becomes arbitrarily large as the transmitter-receiver separation goes to zero. This behavior is more prominent for CSIM in the high-noise regime. With increasing values of transmitter-receiver separation, the gap between packet success probability curves decreases, and the UPM starts to underestimate packet success probability since the multiple-access interference signal PDF becomes heavytailed under the UPM.

For USIM in the low-noise regime, BER is very close to the zero in Fig. 9 for small values of transmitter-receiver separation, which further results in packet success probability being close to 1 under BPM for small values of transmitterreceiver separation in Fig. 10.

Next, we consider the packet success probability in the slow-mobility regime where nodes' locations can be assumed to be static over the course of a packet transmission. Calculations for the slow-mobility regime become a little bit trickier than the fast-mobility regime since bit errors are now dependent due to dependencies among transmitter locations from one bit to another bit.

We begin the slow-mobility regime calculations with CSIM. For CSIM, slowly moving transmitters assumption translates into the fact that the magnitude of the total network interference $|I|$ stays the same during a packet transmission. Therefore, given $|I|=x \geq 0$, and assuming that the background
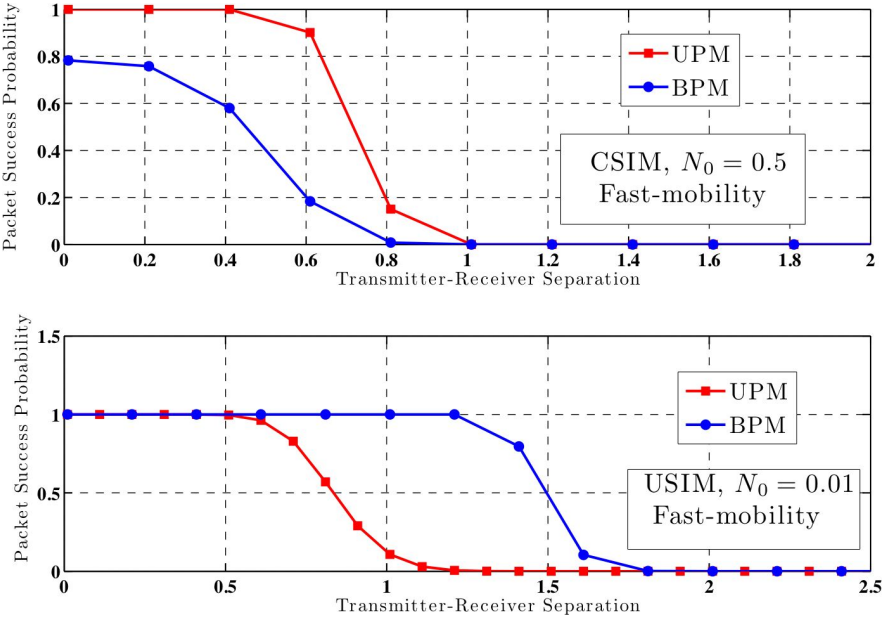

Fig. 10. Change of packet success probability as a function of transmitterreceiver separation in the fast-mobility regime for CSIM and USIM. $D=$ $100, L=10, E_{b}=1, \alpha=6, \lambda=\frac{1}{\pi}$ and $A=5$.

noise impairing transmitted bits is independent from bit to bit, bit errors also become independent. Let $p_{i, \operatorname{CSIM}}(x, d)$ be the probability that a transmitted bit is decoded in error when transmitter-receiver separation is equal to $d,|I|=x$ and $G_{i}$, $i=1,2$, is used to model the signal strength decay. Then,

$$
\begin{aligned}
p_{i, \operatorname{CSIM}}(x, d)= & \frac{1}{4} \operatorname{erfc}\left(\sqrt{\frac{E_{b} G_{i}(d)^{2}}{N_{0}}}+\frac{x}{A \sqrt{N_{0}}}\right) \\
& +\frac{1}{4} \operatorname{erfc}\left(\sqrt{\frac{E_{b} G_{i}(d)^{2}}{N_{0}}}-\frac{x}{A \sqrt{N_{0}}}\right) .
\end{aligned}
$$

Therefore,

$$
\begin{aligned}
& \mathbb{P}(\mathcal{S}(d)|| I \mid=x) \\
& =\sum_{e=0}^{L}\left(\begin{array}{l}
D \\
e
\end{array}\right) p_{i, \operatorname{CSIM}}(x, d)^{e}\left(1-p_{i, \operatorname{CSIM}}(x, d)\right)^{D-e}
\end{aligned}
$$

and

$$
\mathbb{P}(\mathcal{S}(d))=2 \int_{0}^{\infty} \mathbb{P}(\mathcal{S}(d)|| I \mid=x) f_{I}(x) d x
$$

since the PDF of $|I|$ is equal to the twice the positive part of the PDF of $I$.

For USIM, packet success probability calculations become harder since dealing with dependencies among bit errors does not become as easy as in CSIM. However, given the points of the node location process $\mathcal{U}=\left\{X_{i}\right\}_{i=1}^{\infty}$, a reasonable approximation for the multiple access interference under USIM becomes the Gaussian noise approximation with mean zero and variance $Y=\mathbb{E}\left[I^{2} \mid \mathcal{U}\right]$ for $\alpha \in(2,6]$ since the total interference level at the receiver is equal to the summation of large number of small interference signals coming from other transmitters. For larger values of $\alpha$, deviations between the Gaussian noise approximation and the actual interference PDFs become more prominent. Such an approximation becomes better for the BPM since all interference signals are small, and there are no dominant terms. Note that the variance of the Gaussian noise in this approximation is a 

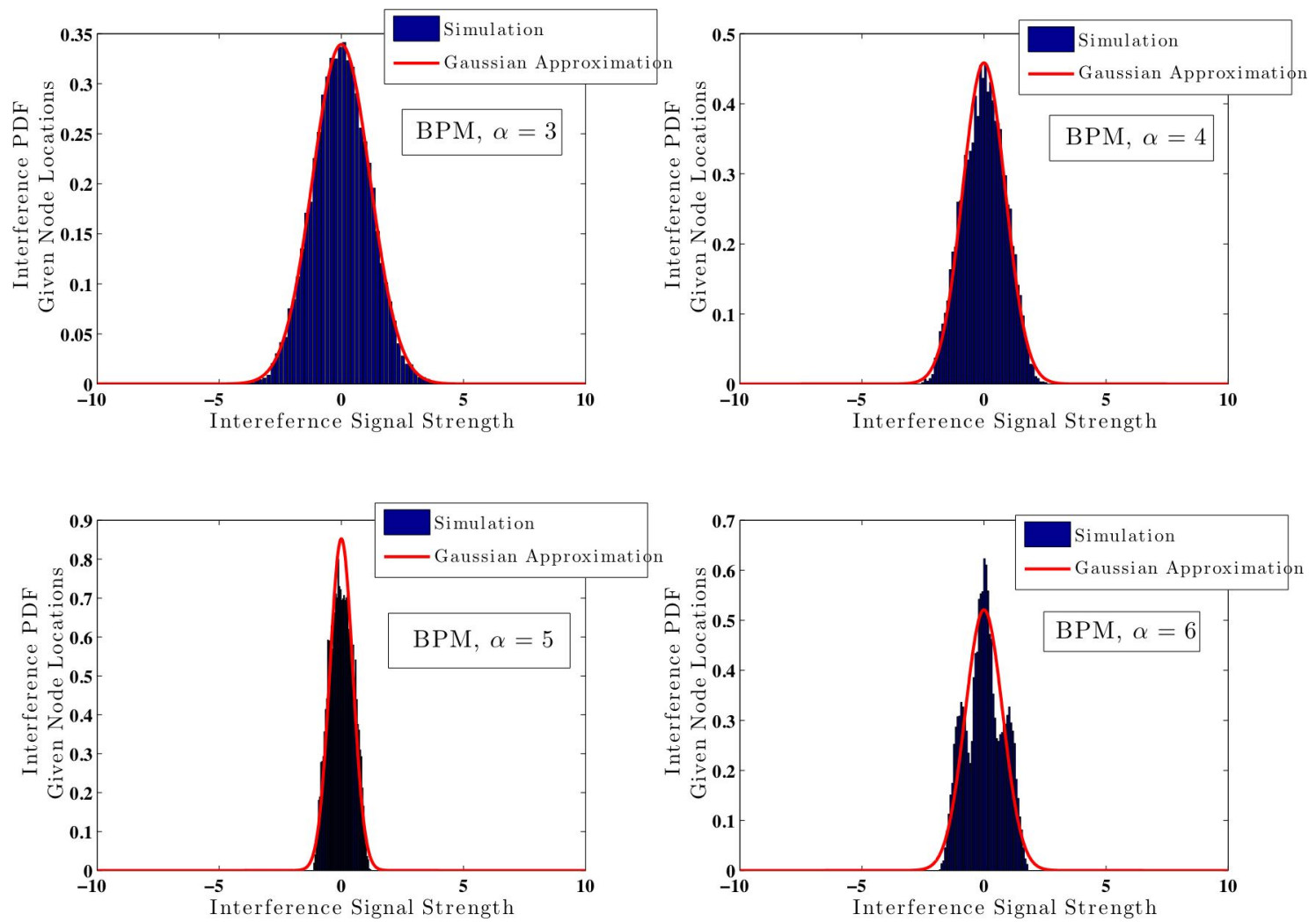

Fig. 11. Approximation of multiple-access interference distribution by means of a Gaussian PDF with mean zero and variance $\mathbb{E}\left[I^{2} \mid \mathcal{U}\right]$ for random realizations of network configurations $\mathcal{U}$ under the BPM and USIM.

random variable, and depends on the particular realization of the network configuration $\mathcal{U}$. Note also for the BPM that

$$
Y=\sum_{i=1}^{\infty} \frac{E_{b}}{\left(1+\left\|X_{i}\right\|^{\frac{\alpha}{2}}\right)^{2}} .
$$

The characteristic function of $Y$ can be obtained exactly by using techniques similar to those of Sections III and IV. However, without going through the similar and tedious calculations, a reasonable approximation for $Y$ is $\tilde{Y}=\sum_{i=1}^{\infty} \frac{E_{b}}{1+\left\|X_{i}\right\|^{\alpha}}$ since for values of $\left\|X_{i}\right\|$ less than 1 , 1 becomes the dominant term in (8), and for the values of $\left\|X_{i}\right\|$ greater than $1,\left\|X_{i}\right\|^{\frac{\alpha}{2}}$ becomes the dominant term in (8). The characteristic function of $\tilde{Y}$ can be readily obtained by replacing $\frac{\alpha}{2}$ with $\alpha$ and removing the operator $\Re(\cdot)$ in Theorem 2. On the other hand, there is no need for such an approximation for the UPM as $Y=\sum_{i=1}^{\infty} \frac{E_{b}}{\left\|X_{i}\right\|-\alpha}$ under the UPM, and its characteristic function can be readily obtained by replacing $\frac{\alpha}{2}$ with $\alpha$ and removing the operator $\Re(\cdot)$ in Theorem 3. Figures 11 and 12 show interference PDFs obtained by means of simulations for given random network configurations $\mathcal{U}$ for both the BPM and the UPM under USIM. We also plot Gaussian approximations with random variance $Y=\mathbb{E}\left[I^{2} \mid \mathcal{U}\right]$ for the multiple-access interference PDF. ${ }^{5}$ We observe fairly good agreement, especially for the BPM, between simulated PDFs and Gaussian approximations in these figures.

Let $Q$ be a Gaussian random variable with mean zero and variance $Y$. Let also $p_{i, \operatorname{USIM}}(x, d)$ be the probability that

\footnotetext{
${ }^{5}$ In fact, we used $\tilde{Y}$ instead of $Y$ while calculating the Gaussian noise variance for the BPM.
}

a transmitted bit is decoded in error when the transmitterreceiver separation is equal to $d, Y=x$ and $G_{i}, i=1,2$, is used to model the signal strength decay. Then,

$$
\begin{aligned}
p_{i, \text { USIM }}(x, d) & =\mathbb{P}\left\{W_{0}+\frac{Q}{A}<-\sqrt{E_{b}} G_{i}(d) \mid Y=x\right\} \\
& =\frac{1}{2} \operatorname{erfc}\left(\sqrt{\frac{E_{b} G_{i}^{2}(d)}{N_{0}+2 \frac{x}{A^{2}}}}\right) .
\end{aligned}
$$

Therefore,

$$
\begin{aligned}
& \mathbb{P}\{\mathcal{S}(d) \mid Y=x\} \\
& \quad=\sum_{e=0}^{L}\left(\begin{array}{c}
D \\
e
\end{array}\right) p_{i, \operatorname{USIM}}(x, d)^{e}\left(1-p_{i, \operatorname{USIM}}(x, d)\right)^{D-e}
\end{aligned}
$$

and

$$
\mathbb{P}\{\mathcal{S}(d)\}=\int_{0}^{\infty} \mathbb{P}\{\mathcal{S}(d) \mid Y=x\} f_{Y}(x) d x,
$$

where $f_{Y}$ is the probability density function of $Y$.

Packet success probability curves under different path-loss and interference models are shown in Fig. 13. We set $\alpha=4$ for USIM since our Gaussian noise approximation is better for values of $\alpha$ around 4 . Since the conditional noise variance coming from other interfering transmitters is divided by $A^{2}$ in (9), we set $A=2$ for USIM to better understand the effect of multiple-access interference on the packet success probability. Qualitative conclusions similar to those in the fast-mobility regime still continue to hold for the slow-mobility regime based on Fig. 13. 

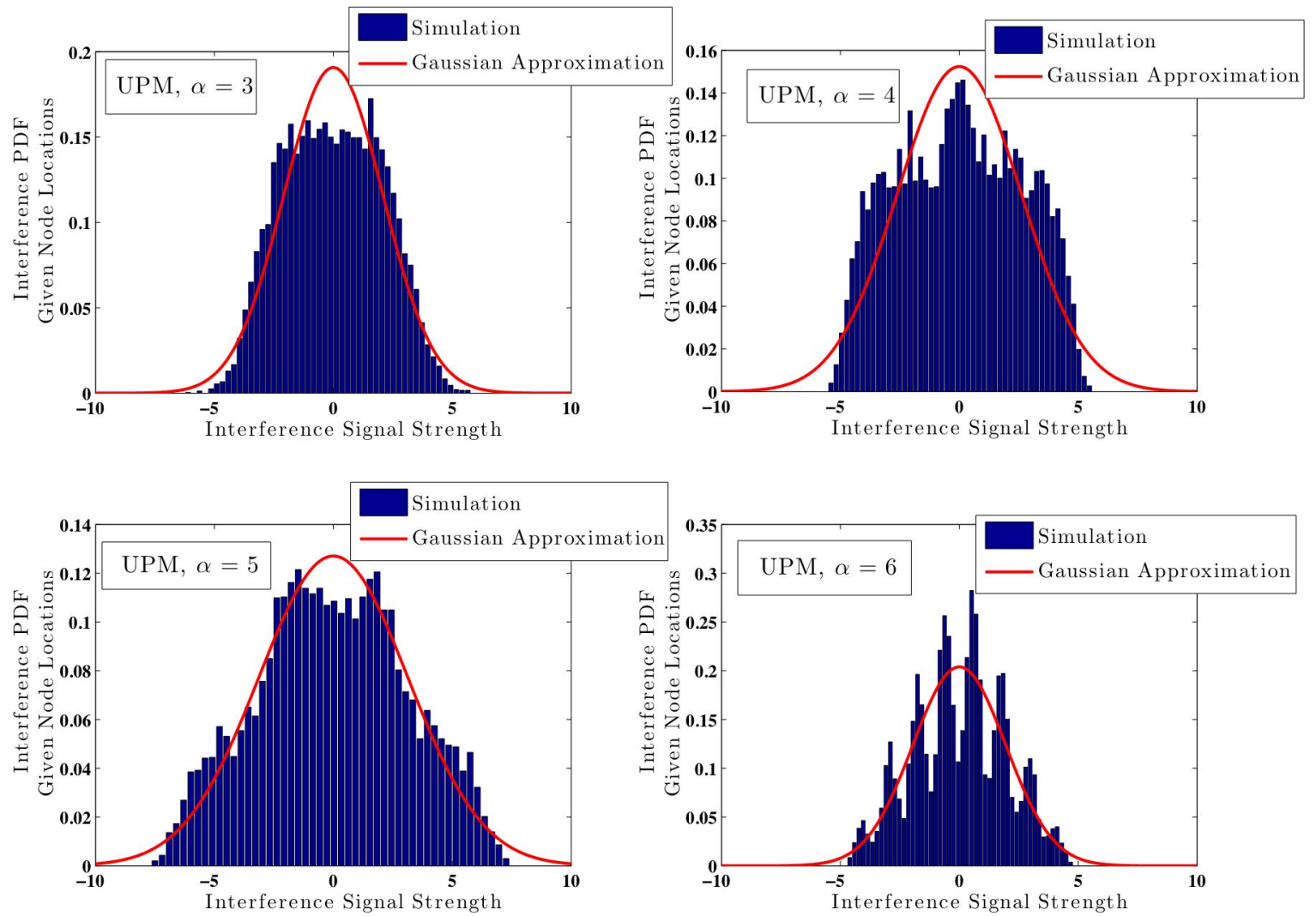

Fig. 12. Approximation of multiple-access interference distribution by means of a Gaussian PDF with mean zero and variance $\mathbb{E}\left[I^{2} \mid \mathcal{U}\right]$ for random realizations of network configurations $\mathcal{U}$ under the UPM and USIM.

The distance range over which packet success probability curves differ from each other significantly is determined by the selection of our parameters. In particular, we have chosen the node density such that a disk with radius 1 [unit distance] contains on the average 1 transmitter. Therefore, if the separation between a transmitter $(\mathrm{Tx})$ and receiver $(\mathrm{Rx})$ pair is equal to 2 [units distance], there are on the average 4 interfering transmitters closer to Rx than Tx. As a result, packet success probability curves under both path-loss models become very close to zero and virtually the same due to these 4 close-by interfering transmitters and the background noise when Tx-Rx separation is larger than 2 [units distance]. On the other hand, if we decrease the node density such that each disk with radius 1 [unit distance] contains 0.1 nodes on the average, the range of distances over which packet success probability curves differ from each other significantly extends to 6-7 [units distance].

\section{Channel Capacity}

Finally, we analyze the information-theoretic wireless channel capacity as a function of the separation between a transmitter-receiver pair. We model the channel between the transmitter and the receiver as a binary symmetric channel with the crossover probability being equal to the probability that a transmitted bit is decoded erroneously at the receiver. We consider both fast-mobility and slow-mobility regimes.

We start with the fast-mobility regime. Let the steady state $\operatorname{BER}_{i}(d), i=1,2$, be the bit error rate calculated in V-A under different interference models when the transmitter-receiver separation is equal to $d . i=1$ corresponds to the UPM, and $i=2$ corresponds to the BPM. Then, the wireless channel capacity $\operatorname{Cap}(d)$ is equal to

$$
\operatorname{Cap}(d)=1-H\left(\operatorname{BER}_{i}(d)\right),
$$

where $H\left(\operatorname{BER}_{i}(d)\right)=-\mathrm{BER}_{i}(d) \log \left(\mathrm{BER}_{i}(d)\right) \quad-$ $\left(1-\operatorname{BER}_{i}(d)\right) \log \left(1-\operatorname{BER}_{i}(d)\right)$.

For the slow-mobility regime, we need to deal with the dependencies among bit errors as it is done in V-B. For CSIM, this is done by conditioning on the interference magnitude $|I|$. By using the same definitions as in V-B, we have

$$
\operatorname{Cap}(d)=1-2 \int_{0}^{\infty} H\left(p_{i, \operatorname{CSIM}}(x, d)\right) f_{I}(x) d x .
$$

For USIM, we eliminate dependencies by first conditioning on the random network configuration $\mathcal{U}$ and then averaging over all possible network configurations. By using the same Gaussion noise approximation and definitions as in V-B, we have

$$
C a p(d)=1-\int_{0}^{\infty} H\left(p_{i, \operatorname{USIM}}(x, d)\right) f_{Y}(x) d x .
$$

Recall that $Y=\mathbb{E}\left[I^{2} \mid \mathcal{U}\right]$, and is determined by random realizations of the network. As we see in Figs. 14 and 15, the deviations between channel capacity curves under bounded and unbounded path-loss models are significant. Qualitative conclusions similar to the ones drawn in previous sections can also be drawn for channel capacity curves. 

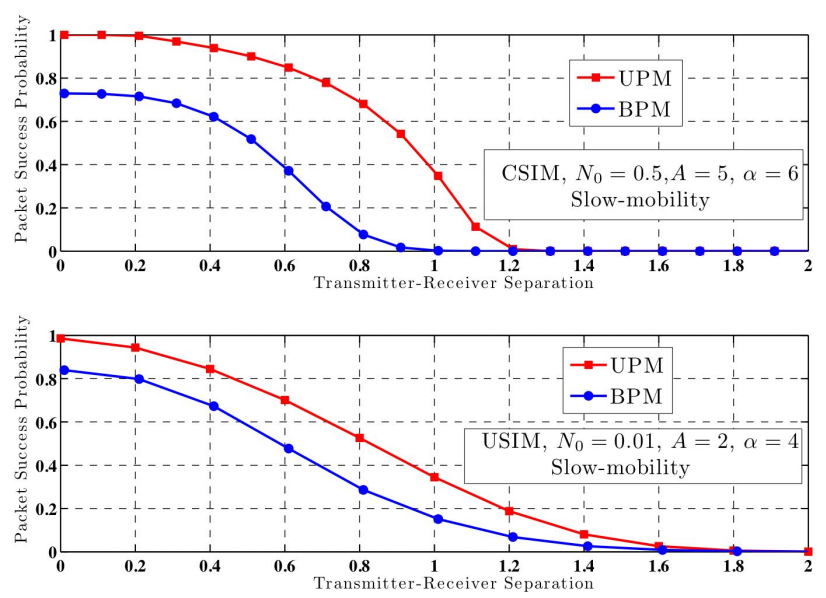

Fig. 13. Change of packet success probability as a function of transmitterreceiver separation in the slow-mobility regime for CSIM and USIM. $D=$ $100, L=10, E_{b}=1, \lambda=\frac{1}{\pi}$.

\section{CONCLUSions}

In this paper, we have examined the effects of the singularity in unbounded path-loss models on network performance. The form $G_{1}(d)=d^{-\frac{\alpha}{2}}$ is used to model the decay of the transmitted signal strength for the unbounded path-loss model, where $d$ is the transmitter-receiver separation and $\alpha>0$ is the path-loss exponent. Therefore, the transmitted signal power decays according to $d^{-\alpha}$. We have compared $G_{1}(d)$ with a bounded signal strength decay function $G_{2}(d)=\frac{1}{1+d^{\frac{\alpha}{2}}}$. $G_{2}$ is a monotonically decreasing function of $d$, it is equal to 1 at $d=0$, and it becomes asymptotically the same with $G_{1}(d)$ as $d \rightarrow \infty$. All of these properties of $G_{2}$ are intuitively pleasing.

We have examined the total network interference signal strength, showing that there is a critical value $\alpha^{*}$ for $\alpha$ at which a phase transition occurs in the behavior of the network interference signal strength. The behavior of the network interference signal strength is similar under the UPM and the BPM for the values of $\alpha$ smaller than $\alpha^{*}$. However, in contrast to the case $\alpha \leq \alpha^{*}$, we have observed a drastic change in the behavior of the network interference signal strength under different path-loss models for the values of $\alpha$ greater than $\alpha^{*}$. In particular, the interference signal strength PDF becomes a heavy-tailed distribution under the UPM. On the contrary, the interference signal strength PDF decays to zero exponentially under the BPM. These results hold for any value of the network node density $\lambda>0$, irrespective of how sparsely or densely populated a network is.

We have also analyzed the effects of the singularity in the UPM for more concrete performance metrics such as bit error rate, packet success probability and wireless channel capacity. We have shown that using the UPM leads to significant deviations from more realistic performance figures obtained by using the BPM.

These results suggest that we should exercise caution when using the classical unbounded path-loss model. The effects of the singularity in the UPM on the network performance cannot be ignored in many situations.
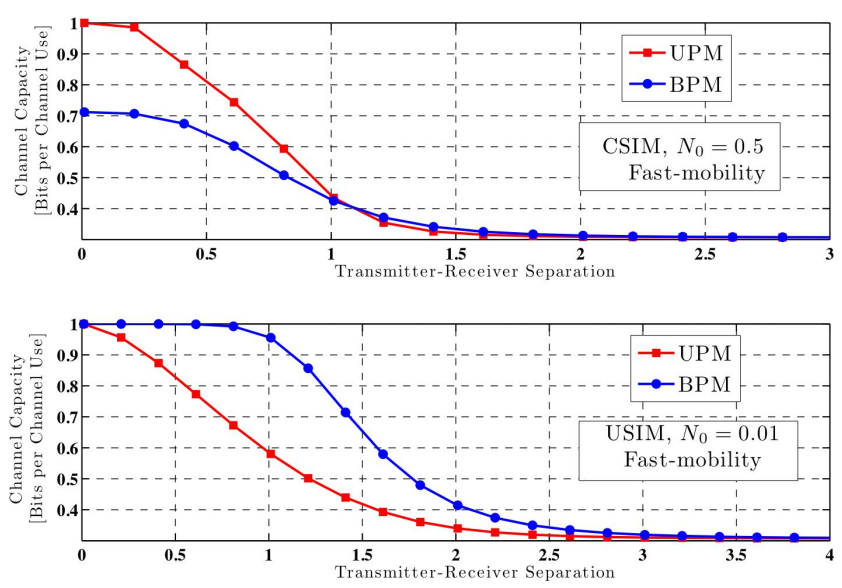

Fig. 14. Change of channel capacity as a function of transmitter-receiver separation in the fast-mobility regime for CSIM and USIM. $E_{b}=1, \alpha=6$, $\lambda=\frac{1}{\pi}$ and $A=5$.
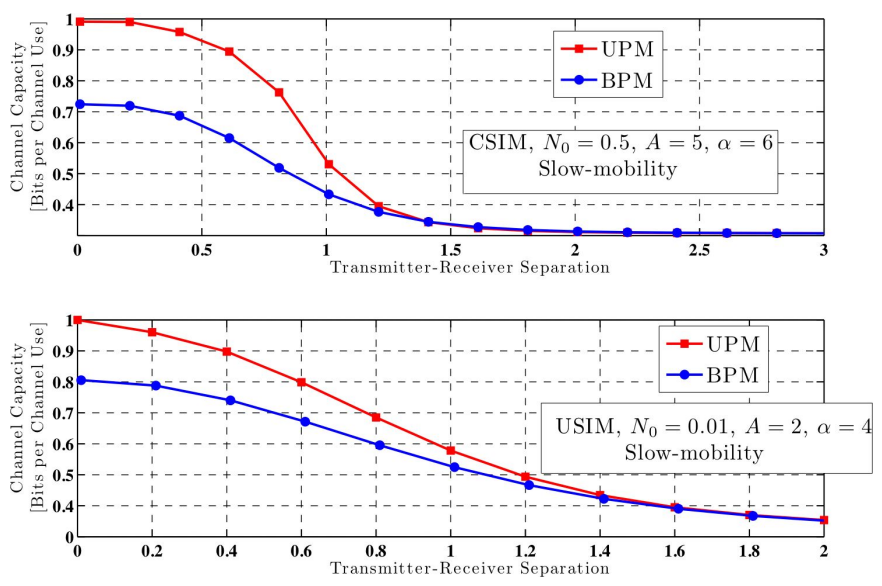

Fig. 15. Change of channel capacity as a function of transmitter-receiver separation in the slow-mobility regime for CSIM and USIM. $E_{b}=1, \lambda=\frac{1}{\pi}$.

\section{APPENDIX A \\ PROOF OF THEOREM 1}

The following weak law for triangular arrays from [16] will be helpful during our calculations.

Lemma 1 (Weak Law for Triangular Arrays): For each $m$, let $Y_{m, k}, 1 \leq k \leq m$, be a set of independent random variables. Let $b_{m}>0$ with $b_{m} \rightarrow \infty$, and let $\bar{Y}_{m, k}=$ $Y_{m, k} \mathbb{1}_{\left\{\left|Y_{m, k}\right| \leq b_{m}\right\}}$. Suppose that $\sum_{k=1}^{m} \mathbb{P}\left\{\left|Y_{m, k}\right|>b_{m}\right\} \rightarrow$ 0 , and $\frac{1}{b_{m}^{2}} \sum_{k=1}^{m} \mathbb{E}\left[\bar{Y}_{m, k}^{2}\right] \rightarrow 0$ as $m \rightarrow \infty$. If we let $S_{m}=Y_{m, 1}+\ldots+Y_{m, m}$ and put $a_{m}=\sum_{k=1}^{m} \mathbb{E}\left[\bar{Y}_{m, k}\right]$, then $\frac{S_{m}-a_{m}}{b_{m}} \stackrel{i . p .}{\rightarrow} 0$.

Proof of Theorem 1: We will give the proof only for the BPM. The proof for the UPM is very similar. Fix the transmitter density $\lambda>0$. Let $\mathcal{B}(\mathbf{0}, 1)$ denote the disk centered around the origin with radius 1 , and let $\left\{X_{k}\right\}_{k \geq 1}$ be a set of independent random variables uniformly distributed over $\mathcal{B}(\mathbf{0}, 1)$. Note that $r X_{k}$ is equal in distribution to $X_{k}^{(r)}$. Then, $I_{r} \stackrel{\mathrm{d}}{=} Z \frac{\sqrt{E_{b}}}{r^{\frac{\alpha}{2}}} \sum_{k=1}^{\left\lceil\lambda \pi r^{2}\right\rceil} \frac{1}{r^{-\frac{\alpha}{2}}+\left\|X_{k}\right\|^{\frac{\alpha}{2}}}$, where $\stackrel{\mathrm{d}}{=}$ means equal in distribution. We will first consider $\alpha<4$. Put $\xi_{r, k}=$ $\frac{1}{r^{-\frac{\alpha}{2}}+\left\|X_{k}\right\|^{\frac{\alpha}{2}}}, \bar{\xi}_{r, k}=\xi_{r, k} \mathbb{1}_{\left\{\xi_{r, k} \leq r^{2}\right\}}$ and $S_{r}=\sum_{k=1}^{\left\lceil\lambda \pi r^{2}\right\rceil} \xi_{r, k}$. We concentrate on $\frac{S_{r}}{r^{2}}$. Observe that $\xi_{r, k} \leq r^{\frac{\alpha}{2}} \leq r^{2}, \forall r \geq 1$. 
Therefore, $\bar{\xi}_{r, k}=\xi_{r, k}$, for all $r \geq 1$ and $1 \leq k \leq\left\lceil\lambda \pi r^{2}\right\rceil$. As a result, $\mathbb{E}\left[\bar{\xi}_{r, k}\right]=\mathbb{E}\left[\xi_{r, k}\right]=\int_{0}^{1} \frac{2 x}{r^{-\frac{\alpha}{2}}+x^{\frac{\alpha}{2}}} d x$. Set $y=x^{2} r^{2}$ and $d y=2 x r^{2} d x$. Then, $\mathbb{E}\left[\xi_{r, k}\right]=r^{\frac{\alpha}{2}-2} \int_{0}^{r^{2}} \frac{1}{1+y^{\frac{\alpha}{4}}} d y$. Now define $f(x)=\int_{0}^{x} \frac{1}{1+y^{\frac{\alpha}{4}}} d y$ and $g(x)=\frac{4}{4-\alpha} x^{1-\frac{\alpha}{4}}$. By L'Hopital's rule, we have $\lim _{x \rightarrow \infty} \frac{f(x)}{g(x)}=1$. Thus, $f(x) \sim$ $\frac{4}{4-\alpha} x^{1-\frac{\alpha}{4}}$ as $x \rightarrow \infty$. This implies that $\int_{0}^{r^{2}} \frac{1}{1+y^{\frac{\alpha}{4}}} d y \sim$ $\frac{4}{4-\alpha} r^{2-\frac{\alpha}{2}}$. Set $a_{r}=\sum_{k=1}^{\left\lceil\lambda \pi r^{2}\right\rceil} \mathbb{E}\left[\xi_{r, k}\right]$. Then, $a_{r} \sim \frac{4}{4-\alpha} \lambda \pi r^{2}$.

We will now check the conditions for triangular arrays.

- $\sum_{k=1}^{\left\lceil\lambda \pi r^{2}\right\rceil} \mathbb{P}\left\{\xi_{r, k}>r^{2}\right\} \rightarrow 0 \quad$ as $\quad r \quad \rightarrow \quad \infty$ : $\mathbb{P}\left\{\xi_{r, k}>r^{2}\right\}=\mathbb{P}\left\{\left\|X_{k}\right\|^{\frac{\alpha}{2}}<r^{-2}-r^{-\frac{\alpha}{2}}\right\}=0$, where the equality follows from the fact that $r^{-\frac{\alpha}{2}}>r^{-2}$ when $r>1$ since $\alpha<4$.

- $\frac{1}{r^{4}} \sum_{k=1}^{\left[\lambda \pi r^{2}\right\rceil} \mathbb{E}\left[\left(\bar{\xi}_{r, k}\right)^{2}\right] \rightarrow 0$ as $r \rightarrow \infty$ :

$$
\mathbb{E}\left[\left(\bar{\xi}_{r, k}\right)^{2}\right] \leq r^{\alpha-2} \int_{0}^{r^{2}} \frac{1}{1+y^{\frac{\alpha}{4}}} d y \sim \frac{4}{4-\alpha} r^{\frac{\alpha}{2}} .
$$

Thus, $\sum_{k=1}^{\left\lceil\lambda \pi r^{2}\right\rceil} \mathbb{E}\left[\left(\bar{\xi}_{r, k}\right)^{2}\right]=\left\lceil\lambda \pi r^{2}\right\rceil \mathbb{E}\left[\left(\bar{\xi}_{r, 1}\right)^{2}\right] \leq$ $\frac{8 \lambda \pi}{4-\alpha} r^{\frac{\alpha}{2}+2}, \quad \forall r \quad$ large enough. Since $\alpha<4$, we have $\quad \lim _{r \rightarrow \infty} \frac{1}{r^{4}} \sum_{k=1}^{\left\lceil\lambda \pi r^{2}\right\rceil} \mathbb{E}\left[\left(\bar{\xi}_{r, k}\right)^{2}\right] \leq$ $\lim _{r \rightarrow \infty} \frac{8 \lambda \pi}{4-\alpha} r^{\frac{\alpha}{2}-2}=0$.

Therefore, the conditions of weak law for triangular arrays are satisfied. We thus have $\frac{S_{r}-a_{r}}{r^{2}} \stackrel{i . p}{\rightarrow} 0$ as $r \rightarrow \infty$. By observing that $I_{r}=Z \frac{\sqrt{E_{b}}}{r^{\frac{\alpha}{2}}} S_{r}$ and $\frac{a_{r}}{r^{2}} \rightarrow \frac{4 \lambda \pi}{4-\alpha}$ as $r \rightarrow \infty$, we conclude that $\frac{I_{r}}{r^{2-\frac{\alpha}{2}}} \stackrel{i . p .}{\rightarrow} Z \frac{4 \sqrt{E_{b}} \lambda \pi}{4-\alpha} \quad$ as $r \rightarrow \infty$. For $\alpha=4$, the same calculations are repeated with $b_{r}=r^{2} \log (r)$.

\section{APPENDIX B}

\section{PROOF OF THEOREM 2}

Similar to the proof of Theorem 1, let $X_{k}^{(r)} \stackrel{\mathrm{d}}{=} r X_{k}$, where the $X_{k}$ 's are independent and uniformly distributed over $\mathcal{B}(\mathbf{0}, 1)$. Then, $I_{r} \stackrel{\mathrm{d}}{=} Z \frac{\sqrt{E_{b}}}{r^{\frac{\alpha}{2}}} \sum_{k=1}^{\left\lceil\lambda \pi r^{2}\right\rceil} \frac{1}{r^{-\frac{\alpha}{2}}+\left\|X_{k}\right\|^{\frac{\alpha}{2}}}$. Let $\xi_{r, k}=\frac{1}{r^{-\frac{\alpha}{2}}+\left\|X_{k}\right\|^{-\frac{\alpha}{2}}}$. Note that $\xi_{r, k} \in\left[\frac{r^{\frac{\alpha}{2}}}{1+r^{\frac{\alpha}{2}}}, r^{\frac{\alpha}{2}}\right]$. Its PDF can be obtained as

$$
f_{r}(x)=\frac{4}{\alpha} \frac{\left(x^{-1}-r^{-\frac{\alpha}{2}}\right)^{\frac{4}{\alpha}-1}}{x^{2}}, x \in\left[\frac{r^{\frac{\alpha}{2}}}{1+r^{\frac{\alpha}{2}}}, r^{\frac{\alpha}{2}}\right] .
$$

Let $\varphi_{r, 1}(t)=\mathbb{E}\left[e^{\dot{n} \xi_{r, 1} t}\right]$. Then, $\varphi_{r, 1}(t)=$ $\frac{4}{\alpha} \int_{\frac{r^{\frac{\alpha}{2}}}{1+r^{\frac{\alpha}{2}}}}^{r^{\frac{\alpha}{2}}} e^{\dot{n} x t} \frac{\left(x^{-1}-r^{-\frac{\alpha}{2}}\right)^{\frac{4}{\alpha}-1}}{x^{2}} d x$. Put $u=x t$. Then, $\varphi_{r, 1}(t)=\frac{4}{\alpha} t \int_{\frac{r^{\frac{\alpha}{2}}}{1+r^{\frac{\alpha}{2}}} t}^{r^{\frac{\alpha}{2}} t} e^{\dot{u} u}\left(\frac{t}{u}-\frac{1}{r^{\frac{\alpha}{2}}}\right)^{\frac{4}{\alpha}-1} u^{-2} d u$.

The characteristic function of the interference $I_{r}$ when the network radius is equal to $r$ is given by

$$
\begin{aligned}
\varphi_{I_{r}}(t) & =\mathbb{E}\left[e^{\left.\dot{u} t Z \frac{\sqrt{E_{b}}}{r^{\frac{\alpha}{2}}} \sum_{k=1}^{\left\lceil\lambda \pi r^{2}\right\rceil} \xi_{r, k}\right]}\right. \\
& =\Re\left(\left[\varphi_{r, 1}\left(t \frac{\sqrt{E_{b}}}{r^{\frac{\alpha}{2}}}\right)\right]^{\left\lceil\lambda \pi r^{2}\right\rceil}\right) .
\end{aligned}
$$

Observe that

$$
\begin{aligned}
1- & \varphi_{r, 1}\left(t \frac{\sqrt{E_{b}}}{r^{\frac{\alpha}{2}}}\right) \\
& =\frac{4}{\alpha} t E_{b}^{\frac{2}{\alpha}} r^{-2} \int_{\frac{t \sqrt{E_{b}}}{1+r^{\frac{\alpha}{2}}}}^{t \sqrt{E_{b}}}\left(1-e^{\dot{a} u}\right)\left(\frac{t}{u}-\frac{1}{\sqrt{E_{b}}}\right)^{\frac{4}{\alpha}-1} u^{-2} d u .
\end{aligned}
$$

Therefore, after some calculus,

$\varphi_{I}(t)=\lim _{r \rightarrow \infty} \varphi_{I_{r}}(t)=\Re\left(\exp \left(-\frac{4 \lambda \pi}{\alpha} E_{b}^{\frac{2}{\alpha}} C(t) t^{\frac{4}{\alpha}}\right)\right)$,

where $C(t)=\int_{0}^{t \sqrt{E_{b}}}(1-\exp (\dot{u} u)) \frac{\left(1-\frac{u}{t \sqrt{E_{b}}}\right)^{\frac{4}{\alpha}-1}}{u^{\frac{4}{\alpha}+1}} d u$.

\section{APPENDIX C}

\section{PROOF OF THEOREM 5}

We will use Tauberian theorems as given in [17] to prove this theorem. A similar result also appeared in [22]. To this end, we first calculate the Laplace transform of $|I|$ on the negative real line.

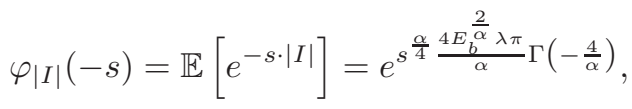

where $s>0$ and $\Gamma(\cdot)$ is the Gamma function. This implies the following relation.

$$
\int_{0}^{\infty} e^{-s \cdot x} \mathbb{P}\{|I|>x\} d x=\frac{1-\varphi_{|I|}(-s)}{s} .
$$

Since $\frac{1-\varphi_{|I|}(-s)}{s} \sim-s^{\frac{4}{\alpha}-1} \frac{4 \Gamma\left(-\frac{4}{\alpha}\right) E_{b}^{\frac{2}{\alpha}} \lambda \pi}{\alpha}$ as $s \rightarrow 0$, we have $\mathbb{P}\{|I|>x\} \sim \lambda \pi E_{b}^{\frac{2}{\alpha}} x^{-\frac{4}{\alpha}}$ as $x \rightarrow \infty$ by using the Theorem 4 of Chapter 13 in [17] and the relation $\Gamma(z)=(z-1) \Gamma(z-1)$.

\section{APPENDIX D \\ PROOF OF THEOREM 6}

We use the following result from [16].

Lemma 2 (The Lindeberg-Feller Theorem): For each $m$, let $Y_{m, k}, 1 \leq k \leq m$, be independent random variables with $\mathbb{E}\left[Y_{m, k}\right]=0$. Suppose that $\sum_{k=1}^{m} \mathbb{E}\left[\left|Y_{m, k}\right|^{2}\right] \rightarrow \sigma^{2}>0$, and $\forall \epsilon>0, \lim _{m \rightarrow \infty} \sum_{k=1}^{m} \mathbb{E}\left[\left|Y_{m, k}\right|^{2} ;\left|Y_{m, k}\right|>\epsilon\right]=0$. Then, $S_{m}=\sum_{k=1}^{m} Y_{m, k} \Rightarrow \mathcal{N}\left(0, \sigma^{2}\right)$ as $m \rightarrow \infty$.

Proof of Theorem 6: We will give the proof only for the BPM. The proof for the UPM is very similar. Fix the transmitter density $\lambda>0$. With the same definitions of the proof of Theorem 1, we have $I_{r} \stackrel{\mathrm{d}}{=} \frac{\sqrt{E_{b}}}{r^{\frac{\alpha}{2}}} \sum_{k=1}^{\left\lceil\lambda \pi r^{2}\right\rceil} \frac{Z_{k}}{r^{-\frac{\alpha}{2}}+\left\|X_{k}\right\|^{\frac{\alpha}{2}}}$. We will first consider $\alpha<2$. Let $\xi_{r, k}=\frac{Z_{k} \sqrt{E_{b}}}{r^{-\frac{\alpha}{2}}+\left\|X_{k}\right\|^{\frac{\alpha}{2}}}$ and $S_{r}=\sum_{k=1}^{\left\lceil\lambda \pi r^{2}\right\rceil} \xi_{r, k}$. We concentrate on $\frac{S_{r}}{r}$, and check the conditions for the Lindeberg-Feller theorem. We only check the first condition. The second one is automatically satisfied since $\left|\xi_{r, k}\right| \leq \sqrt{E_{b}} r^{\frac{\alpha}{2}}$ and $\alpha<2$. We have $\sum_{k=1}^{\left\lceil\lambda \pi r^{2}\right\rceil} \mathbb{E}\left[\left|\frac{1}{r} \xi_{r, k}\right|^{2}\right]=\frac{2 E_{b}\left\lceil\lambda \pi r^{2}\right\rceil}{r^{2}} \int_{0}^{1} \frac{x}{\left(r^{-\frac{\alpha}{2}}+x^{\frac{\alpha}{2}}\right)^{2}} d x$, so we wish to analyze $\lim _{r \rightarrow \infty} \int_{0}^{1} \frac{x}{\left(r^{-\frac{\alpha}{2}}+x^{\frac{\alpha}{2}}\right)^{2}}$. Note that 
$\frac{x}{\left(r^{-\frac{\alpha}{2}}+x^{\frac{\alpha}{2}}\right)^{2}} \leq x^{1-\alpha}$ and $\int_{0}^{1} x^{1-\alpha} d x=\frac{1}{2-\alpha}<$ $\infty$. By using the dominated convergence theorem [19], we have $\lim _{r \rightarrow \infty} \int_{0}^{1} \frac{x}{\left(r^{-\frac{\alpha}{2}}+x^{\frac{\alpha}{2}}\right)^{2}}=\frac{1}{2-\alpha}$, which implies $\lim _{r \rightarrow \infty} \sum_{k=1}^{\left\lceil\lambda \pi r^{2}\right\rceil} \mathbb{E}\left[\left|\frac{1}{r} \xi_{r, k}\right|^{2}\right]=\frac{2 \lambda \pi E_{b}}{2-\alpha}$. Therefore, $\frac{S_{r}}{r} \Rightarrow$ $\mathcal{N}\left(0, \frac{2 \lambda \pi E_{b}}{2-\alpha}\right)$, which implies $\frac{I_{r}}{r^{\frac{2-\alpha}{2}}} \Rightarrow \mathcal{N}\left(0, \frac{2 \lambda \pi E_{b}}{2-\alpha}\right)$.

For $\alpha=2$, similar calculations are repeated with $\frac{S_{r}}{r \sqrt{\log (r)}}$.

\section{APPENDIX E \\ PROOF OF THEOREM 9}

We focus on the Laplace transforms on the positive real line. Our proof depends on the following lemma.

Lemma 3: Let $X$ be a random variable such that $\varphi_{X}(s)=$ $\mathbb{E}\left[e^{s X}\right]<\infty$ for some $s>0$. Then, $\mathbb{P}\{X>x\} \leq$ $\varphi_{X}(s) e^{-s x}$.

Proof: $e^{s x} \mathbb{1}_{\{X>x\}} \leq e^{s X} \mathbb{1}_{\{X>x\}} \leq e^{s X}$. Thus, $e^{s x} \mathbb{P}\{X>x\} \leq \mathbb{E}\left[e^{s X}\right]$. As a result, $\mathbb{P}\{X>x\} \leq$ $e^{-s x} \varphi_{X}(s)$.

We will show that $\lim _{r \rightarrow \infty} \varphi_{I_{r}}(s)=\varphi_{I}(s)<\infty$ for all $s>0$. This implies that $\log (\mathbb{P}\{I>x\}) \leq-s x+\log \left(\varphi_{I}(s)\right)$. Therefore, $\lim _{x \rightarrow \infty}\left|\frac{\log (\mathbb{P}\{I>x\})}{x}\right| \geq s$. This concludes the proof since $s$ is arbitrary and $I$ has a symmetric distribution around 0 . For the rest of the proof, we will focus on showing $\lim _{r \rightarrow \infty} \varphi_{I_{r}}(s)=\varphi_{I}(s)<\infty$ for all $s>0$.

When the network radius is equal to $r>0$, the demodulated interference signal coming from the $k^{\text {th }}$ interferer is given by $I_{k}^{(r)}=Z_{k} \cos \left(\phi_{k}\right) \gamma_{k} \sqrt{E_{b}} G_{B}\left(\left\|X_{k}^{(r)}\right\|\right)$, where $\phi_{k}$ is the random phase shift and $\gamma_{k}$ is the fading coefficient for the $k^{\text {th }}$ interference signal. Then, $I_{r}=\sum_{k=1}^{\left\lceil\lambda \pi r^{2}\right\rceil} I_{k_{2}}^{(r)}$. Let $\varphi_{r, 1}(s)=\mathbb{E}\left[e^{s \cdot I_{1}^{(r)}}\right]$. Then, $\varphi_{I_{r}}(s)=\left(\varphi_{r, 1}(s)\right)^{\left\lceil\lambda \pi r^{2}\right\rceil}$. We will show that $\varphi_{r, 1}(s)=1+O\left(r^{-2}\right)$ for any given $s$, which implies $\varphi_{I}(s)<\infty$.

Let $\beta_{1}=Z_{1} \cos \left(\phi_{1}\right)$. Then, $\beta_{1}$ has a symmetric distribution on $[-1,1]$, which is symmetric around 0 . Let $p$ be the distribution of $\beta_{1}$. We can write $\varphi_{r, 1}(s)$ as follows.

$$
\begin{aligned}
\varphi_{r, 1}(s)=\int_{0}^{1} p(y) \mathbb{E}[ & \exp \left(s \cdot y \gamma_{1} \sqrt{E_{b}} G_{B}\left(\left\|X_{1}^{(r)}\right\|\right)\right) \\
+ & \left.\exp \left(-s \cdot y \gamma_{1} \sqrt{E_{b}} G_{B}\left(\left\|X_{1}^{(r)}\right\|\right)\right)\right] d y .
\end{aligned}
$$

Let $Y(r, y)=\mathbb{E}\left[\exp \left(s \cdot y \gamma_{1} \sqrt{E_{b}} G_{B}\left(\left\|X_{1}^{(r)}\right\|\right)\right)\right.$ $\left.+\exp \left(-s \cdot y \gamma_{1} \sqrt{E_{b}} G_{B}\left(\left\|X_{1}^{(r)}\right\|\right)\right)\right]$ and $q$ be the probability distribution of $\gamma_{1}$. Then,

$$
\begin{aligned}
Y(r, y)=\frac{2}{r^{2}} \int_{0}^{\infty} q(a) & \int_{0}^{r} \rho\left(\exp \left(s \cdot y a \sqrt{E_{b}} G_{B}(\rho)\right)\right. \\
& \left.+\exp \left(-s \cdot y a \sqrt{E_{b}} G_{B}(\rho)\right)\right) d \rho d a .
\end{aligned}
$$

We first focus on inner integral over $\rho$. Let

$$
\begin{aligned}
A(r, y, a)=\int_{0}^{r} \rho(\exp (s & \left.\cdot y a \sqrt{E_{b}} G_{B}(\rho)\right) \\
& \left.+\exp \left(-s \cdot y a \sqrt{E_{b}} G_{B}(\rho)\right)\right) d \rho .
\end{aligned}
$$

Using integration by parts with $u=$ $\exp \left(s \cdot y a \sqrt{E_{b}} G_{B}(\rho)\right)+\exp \left(-s \cdot y a \sqrt{E_{b}} G_{B}(\rho)\right)$ and $d v=\rho d \rho$, we have

$$
\begin{array}{r}
A(r, y, a)=\frac{r^{2}}{2}\left(\exp \left(s \cdot y a \sqrt{E_{b}} G_{B}(r)\right)\right. \\
\left.+\exp \left(-s \cdot y a \sqrt{E_{b}} G_{B}(r)\right)\right) \\
-\frac{s \cdot y a \sqrt{E_{b}}}{2} \int_{0}^{r} \rho^{2} G_{B}^{\prime}(\rho)\left(\exp \left(s \cdot y a \sqrt{E_{b}} G_{B}(\rho)\right)\right. \\
\left.-\exp \left(-s \cdot y a \sqrt{E_{b}} G_{B}(\rho)\right)\right) d \rho .
\end{array}
$$

Therefore,

$$
\begin{aligned}
& \varphi_{r, 1}(s)=\int_{0}^{1} p(y) Y(r, y) d y \\
& =\int_{0}^{1} \int_{0}^{\infty} p(y) q(a)\left(e^{s \cdot y a \sqrt{E_{b}} G_{B}(r)}+e^{-s \cdot y a \sqrt{E_{b}} G_{B}(r)}\right) d a d y \\
& -\frac{s \sqrt{E_{b}}}{r^{2}} \int_{0}^{1} \int_{0}^{\infty} \int_{0}^{r} p(y) q(a) y a \rho^{2} G_{B}^{\prime}(\rho) \\
& \cdot\left(e^{s \cdot y a \sqrt{E_{b}} G_{B}(\rho)}-e^{-s \cdot y a \sqrt{E_{b}} G_{B}(\rho)}\right) d \rho d a d y .
\end{aligned}
$$

Let

$$
\begin{aligned}
B_{1}(r)=\int_{0}^{1} \int_{0}^{\infty} & p(y) q(a) \\
& \cdot\left(e^{s \cdot y a \sqrt{E_{b}} G_{B}(r)}+e^{-s \cdot y a \sqrt{E_{b}} G_{B}(r)}\right) d a d y
\end{aligned}
$$

and

$$
\begin{aligned}
B_{2}(r)=\frac{s \sqrt{E_{b}}}{r^{2}} & \int_{0}^{1} \int_{0}^{\infty} \int_{0}^{r} p(y) q(a) y a \rho^{2} G_{B}^{\prime}(\rho) \\
& \cdot\left(e^{s \cdot y a \sqrt{E_{b}} G_{B}(\rho)}-e^{-s \cdot y a \sqrt{E_{b}} G_{B}(\rho)}\right) d \rho d a d y .
\end{aligned}
$$

We first show that $B_{1}(r)=1+O\left(r^{-\alpha}\right)$. By using Taylor series expansion for exponential functions, we have

$$
\begin{aligned}
& B_{1}(r) \\
& =1+\sum_{k=1}^{\infty} \int_{0}^{1} \int_{0}^{\infty} 2 p(y) q(a) \frac{\left(s \cdot y a \sqrt{E_{b}} G_{B}(r)\right)^{2 k}}{(2 k) !} d a d y .
\end{aligned}
$$

Let $S=\sum_{k=1}^{\infty} \int_{0}^{1} \int_{0}^{\infty} 2 p(y) q(a) \frac{\left(s \cdot y a \sqrt{E_{b}} G_{B}(r)\right)^{2 k}}{(2 k) !} d a d y$. Then, for $r$ large enough,

$$
\begin{aligned}
S & \leq 2 \sum_{k=1}^{\infty} \int_{0}^{\infty} q(a) \frac{\left(s \cdot a r^{-\frac{\alpha}{2}} \sqrt{E_{b}}\right)^{2 k}}{(2 k) !} d a \\
& \leq 2 r^{-\alpha} \mathbb{E}\left[\exp \left(s \cdot \gamma_{1} \sqrt{E_{b}}\right)\right]<\infty
\end{aligned}
$$

Thus, $B_{1}(r)=1+O\left(r^{-\alpha}\right)$. We divide $B_{2}(r)$ into two pieces $B_{3}(r)$ and $B_{4}(r) . B_{3}(r)$ equals to

$$
\begin{aligned}
B_{3}(r)=\frac{s \sqrt{E_{b}}}{r^{2}} \int_{0}^{1} \int_{0}^{\infty} \int_{0}^{T} p(y) q(a) y a \rho^{2} G_{B}^{\prime}(\rho) \\
\cdot\left(e^{s \cdot y a \sqrt{E_{b}} G_{B}(\rho)}-e^{-s \cdot y a \sqrt{E_{b}} G_{B}(\rho)}\right) d \rho d a d y .
\end{aligned}
$$


We show that $B_{3}(r)=O\left(r^{-2}\right)$ as $r \rightarrow \infty$.

$$
\begin{aligned}
& \left|B_{3}(r)\right| \leq \frac{s \sqrt{E_{b}} T^{2}}{r^{2}} \int_{0}^{\infty} q(a) a e^{s \cdot a \sqrt{E_{b}} G_{B}(0)} d a \\
& \cdot\left(-\int_{0}^{T} G_{B}^{\prime}(\rho) d \rho\right) \\
& =\frac{s \cdot\left(G_{B}(0)-G_{B}(T)\right) \sqrt{E_{b}} T^{2}}{r^{2}} \mathbb{E}\left[\gamma_{1} e^{s \cdot \gamma_{1} \sqrt{E_{b}} G_{B}(0)}\right]<\infty .
\end{aligned}
$$

$B_{4}(r)$ equals to

$$
\begin{aligned}
B_{4}(r)=\frac{s \sqrt{E_{b}}}{r^{2}} & \int_{0}^{1} \int_{0}^{\infty} \int_{T}^{r} p(y) q(a) y a \rho^{2} G_{B}^{\prime}(\rho) \\
& \cdot\left(e^{s \cdot y a \sqrt{E_{b}} G_{B}(\rho)}-e^{-s \cdot y a \sqrt{E_{b}} G_{B}(\rho)}\right) d \rho d a d y .
\end{aligned}
$$

We now show that $B_{4}(r)=O\left(r^{-2}\right)$ as $r \rightarrow \infty$. Let $u=$ $G_{B}(\rho)$. Then,

$$
\begin{aligned}
&\left|B_{4}(r)\right| \leq \frac{s \cdot \sqrt{E_{b}}}{r^{2}} \int_{0}^{1} \int_{0}^{\infty} \int_{0}^{G_{B}(T)} p(y) q(a) y a\left(G_{B}^{-1}(u)\right)^{2} \\
& \cdot\left(e^{s \cdot y a \sqrt{E_{b}} u}-e^{\left.-s \cdot y a \sqrt{E_{b}} u\right)}\right) d u d a d y .
\end{aligned}
$$

We need the following lemma about the behavior of $G_{B}^{-1}(u)$ near the origin.

Lemma 4: There exists $c>0$ and $\epsilon>0$ such that $G_{B}^{-1}(u) \leq c \cdot u^{-\frac{2}{\alpha}}$ for all $u \in(0, \epsilon)$.

Proof: Since $G_{B}(d) \sim d^{-\frac{\alpha}{2}}$ as $d \rightarrow \infty$, for any $c_{1}>$ 1 , we have $G_{B}\left(d^{\frac{2}{\alpha}}\right) \leq c_{1} d^{-1}$ for all $d$ large enough. Since $G_{B}$ is a non-increasing function, $G_{B}^{-1}$ also becomes a nonincreasing function. Therefore, $G_{B}^{-1}\left(G_{B}\left(d^{\frac{2}{\alpha}}\right)\right)=d^{\frac{2}{\alpha}}$, and $G_{B}^{-1}\left(c_{1} d^{-1}\right) \leq d^{\frac{2}{\alpha}}$ for all $d$ large enough. As a result, we can find a large enough constant $M>0$ such that $G_{B}^{-1}\left(c_{1} d^{-1}\right) \leq$ $d^{\frac{2}{\alpha}}$ for all $d \geq M$. Put $u=c_{1} d^{-1}$. Then, $G_{B}^{-1}(u) \leq c u^{-\frac{2}{\alpha}}$ for all $u \in\left(0, \frac{c_{1}}{M}\right)$, where $c=c_{1}^{\frac{2}{\alpha}}$.

We now divide the inner most integral in (18) into two pieces and bound them by using Lemma 4 and $\alpha>2$ as follows.

$$
\begin{aligned}
& \left|B_{4}(r)\right| \leq \frac{s \cdot \sqrt{E_{b}}}{r^{2}} \int_{0}^{1} \int_{0}^{\infty} \int_{0}^{\epsilon} p(y) q(a) y a\left(G_{B}^{-1}(u)\right)^{2} \\
& \cdot\left(e^{s \cdot y a \sqrt{E_{b}} u}-e^{\left.-s \cdot y a \sqrt{E_{b}} u\right)}\right) d u d a d y \\
& +\frac{s \cdot \sqrt{E_{b}}}{r^{2}} \int_{0}^{1} \int_{0}^{\infty} \int_{\epsilon}^{G_{B}(T)} p(y) q(a) y a\left(G_{B}^{-1}(u)\right)^{2} \\
& \cdot\left(e^{s \cdot y a \sqrt{E_{b}} u}-e^{\left.-s \cdot y a \sqrt{E_{b}} u\right)}\right) d u d a d y \\
& \leq \frac{s \cdot \sqrt{E_{b}}}{r^{2}}\left(c_{1} \int_{0}^{\infty} \int_{0}^{\epsilon} q(a) a u^{-\frac{4}{\alpha}} u e^{s \cdot a \epsilon \sqrt{E_{b}}} d u d a\right. \\
& \left.+c_{2} \int_{0}^{\infty} q(a) a e^{s \cdot a \sqrt{E_{b}} G_{B}(T)} d a\right) \\
& =\frac{s \cdot \sqrt{E_{b}}}{r^{2}}\left(c_{1} \mathbb{E}\left[\gamma_{1} e^{s \cdot \gamma_{1} \epsilon \sqrt{E_{b}}}\right]\left(\epsilon^{\frac{-4}{\alpha}+2}\right)\left(\frac{-4}{\alpha}+2\right)^{-1}\right. \\
& \left.+c_{2} \mathbb{E}\left[\gamma_{1} e^{s \cdot \gamma_{1} \sqrt{E_{b}} G_{B}(T)}\right]\right)<\infty,
\end{aligned}
$$

where $c_{2}=\left(G_{B}^{-1}(\epsilon)\right)^{2}\left(G_{B}(T)-G_{B}(\epsilon)\right)$. Thus, $B_{2}(r)=$ $B_{3}(r)+B_{4}(r)=O\left(r^{-2}\right)$. This concludes the proof as $\varphi_{r, 1}(s)=B_{1}(r)-B_{2}(r)=1+O\left(r^{-\alpha}\right)+O\left(r^{-2}\right)=$ $1+O\left(r^{-2}\right)$ since $\alpha>2$.

\section{APPENDIX F}

\section{PROOF OF THEOREM 10}

We will use direct calculations by using the interference characteristic function given in Theorem 8 to estimate $\mathbb{P}\{|I|>x\}$ since it is hard to obtain the Laplace transform for $|I|$ under USIM to use Tauberian theorems in [17]. Let $C_{1}=\frac{4 \lambda \pi}{\alpha} E_{b}^{\frac{2}{\alpha}} C$, where $C$ is given as in Theorem 8. After some calculus with change of variables $u=t x$, one obtains,

$$
\begin{aligned}
& \mathbb{P}\{|I|>x\}=1-\frac{1}{\pi} \int_{0}^{x} \int_{-\infty}^{\infty} e^{-\dot{x} t y} \varphi_{I}(t) d t d y \\
& =\frac{2}{\pi} C_{1} x^{\frac{-4}{\alpha}} \int_{0}^{x \cdot C_{1}^{\frac{-\alpha}{4}}} \sum_{k=1}^{\infty} \frac{\sin (u)}{u} u^{\frac{4}{\alpha}} \frac{\left(-C_{1} u^{\frac{4}{\alpha}} x^{\frac{-4}{\alpha}}\right)^{k-1}}{k !} d u \\
& +\frac{2}{\pi} C_{1} x^{\frac{-4}{\alpha}} R(x),
\end{aligned}
$$

where

$R(x)=\int_{x \cdot C_{1}^{\frac{-\alpha}{4}}}^{\infty} \sum_{k=1}^{\infty} \frac{\sin (u)}{u} u^{\frac{4}{\alpha}} \frac{(-1)^{k-1}\left(C_{1} u^{\frac{4}{\alpha}} x^{\frac{-4}{\alpha}}\right)^{k-1}}{k !} d u$.

Observe that $R(x)=o(1)$ as $x \rightarrow \infty$. We need the following lemma to conclude the proof.

Lemma 5: Let $a \in(0,1]$. Then,

$$
Z=\sum_{k=1}^{\infty}(-1)^{k-1} \frac{a^{k-1}}{k !} \geq \exp (-a) \text {. }
$$

Proof: $Z=1-a\left(\frac{1}{2 !}-\frac{a}{3 !}\right)-a^{3}\left(\frac{1}{4 !}-\frac{a}{5 !}\right)-$ $a^{5}\left(\frac{1}{6 !}-\frac{a}{7 !}\right) \cdots$. For $k \geq 2$, let us compare $\frac{1}{(k-1) !}-\frac{a}{k !}$ with $\frac{1}{k !}-\frac{a}{(k+1) !} \cdot\left(\frac{1}{(k-1) !}-\frac{a}{k !}\right)-\left(\frac{1}{k !}-\frac{a}{(k+1) !}\right)=\frac{k^{2}-1-a k}{(k+1) !} \geq$ 0 for $k \geq 2$. Thus, $Z \geq 1-a\left(\frac{1}{1 !}-\frac{a}{2 !}\right)-a^{3}\left(\frac{1}{3 !}-\frac{a}{4 !}\right)-$ $a^{5}\left(\frac{1}{5 !}-\frac{a}{6 !}\right) \cdots=e^{-a}$.

Therefore,

$$
\begin{aligned}
& \mathbb{P}\{|I|>x\} \\
& \quad \geq \frac{2}{\pi} C_{1} x^{\frac{-4}{\alpha}} \int_{0}^{x \cdot C_{1}^{\frac{-\alpha}{4}}} \frac{\sin (u)}{u} u^{\frac{4}{\alpha}} e^{-C_{1} x^{\frac{-4}{\alpha}} u^{\frac{4}{\alpha}}} d u+o\left(x^{\frac{-4}{\alpha}}\right) \\
& \geq K x^{\frac{-4}{\alpha}}, \text { for some } K>0 .
\end{aligned}
$$

\section{REFERENCES}

[1] T. S. Rappaport, Wireless Communications: Principles and Practice, Prentice Hall, Upper Saddle River, NJ, 1999.

[2] S. B. Lowen and M. C. Teich, "Power-law shot noise," IEEE Trans. Inform. Theory, vol. IT-36, no. 6, pp. 1302-1318, Nov. 1990.

[3] E. S. Sousa and J. A. Silvester, "Optimum transmission ranges in a direct sequence spread spectrum multihop packet radio network," IEEE J. Select. Areas Commun., vol. 8, no. 5, pp. 762-771, June 1990.

[4] E. S. Sousa, "Performance of a spread spectrum packet radio network in a Poisson field of interferers," IEEE Trans. Inform. Theory, vol. 38, no. 6, pp. 1743-1754, November 1992.

[5] C. C. Chan and V. H. Stephen, "Calculating the outage probability in a CDMA network with spatial Poisson traffic," IEEE Trans. Veh. Technol., vol. 50, no. 1, pp. 183-204, Jan. 2001.

[6] P. C. Pinto and M. Z. Win, "Communication in a poisson field of interferers," Proc. 40th Annual Conference on Information Sciences and Systems, Princeton, NJ, March 2006.

[7] S. Weber and J. G. Andrews, "Bounds on the SIR distribution for a class of channel models in ad hoc networks," Proc. 49th Annual IEEE Globecom Conference, San Francisco, CA, November 2006. 
[8] F. Baccelli and B. Błaszczyszyn, "On a coverage process ranging from the Boolean model to the Poisson Voronoi tessellation with applications to wireless communications," Adv. Appl. Prob., vol. 33, no. 2, pp. 293-323, 2001.

[9] O. Dousse and P. Thiran, "Connectivity vs capacity in dense ad hoc networks," Proc. Twenty Third Annual Joint Conference of the IEEE Computer and Communications Societies, vol. 1, pp. 476-486, Hong Kong, China, March 2004.

[10] O. Arpacioglu and Z. J. Haas, "On the scalability and capacity of wireless networks with omnidirectional antennas," Proc. Third International Symposium on Information Processing in Sensor Networks, pp. 169-177, Berkeley, CA, April 2004.

[11] R. K. Ganti and M. Haenggi, "Interference and outage in clustered wireless ad hoc networks," IEEE Trans. Inform. Theory, accepted, June 2008.

[12] J. F. C. Kingman, Poisson Processes, Clarendon Press, Oxford, UK, 1993.

[13] A. Ozgur, O. Leveque and D. N. C. Tse, "Hierarchical cooperation achieves optimal capacity scaling in ad hoc networks," IEEE Trans. Inform. Theory, vol. 53, no. 10, pp. 3549-3572, October 2007.

[14] U. Niesen, P. Gupta and D. Shah, "The capacity region of large wireless networks," Allerton Conference on Communication, Control and Computing, Allerton, IL, September 2008.

[15] D. J. Daley, "The definition of a multi-dimensional generalization of shot noise," J. Appl. Prob., vol. 8, no. 1, pp. 128-135, March 1971.

[16] R. Durrett, Probability: Theory and Examples, Duxbury Press, Belmont, CA, second edition, 1996.

[17] W. Feller, An Introduction to Probability Theory and Its Applications, John Wiley and Sons, Inc., New York, vol. 2, second edition, 1970.

[18] H. Inaltekin, Topics on Wireless Network Design: Game Theoretic MAC Protocol Design and Interference Analysis for Wireless Networks, $\mathrm{PhD}$ Dissertation, School of Electrical and Computer Engineering, Cornell University, Ithaca, NY, Aug. 2006.

[19] W. Rudin, Real and Complex Analysis, McGraw-Hill, New York, third edition, 1987.

[20] D. Tse and P. Viswanath, Fundamentals of Wireless Communications, Cambridge University Press, New York, 2005.

[21] S. B. Wicker, Error Control Systems for Digital Communication and Storage, Prentice Hall, Upper Saddle River, NJ, 1995.

[22] C. Adjih, E. Baccelli, T.Clausen, P. Jacquet and R. Rodolakis, "Fish eye OLSR scaling properties," IEEE J. Communication and Networks, vol. 6, no. 4, pp. 343-351, December 2004.

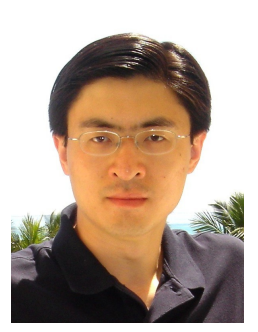

Mung Chiang (S'00, M'03, SM'08) is an Associate Professor of Electrical Engineering, and an Affiliated Faculty of Applied and Computational Mathematics and of Computer Science, at Princeton University. He received the B.S. (Honors) in Electrical Engineering and Mathematics, M.S. and Ph.D. degrees in Electrical Engineering from Stanford University in 1999, 2000, and 2003, respectively, and was an Assistant Professor at Princeton University 2003-2008. His research areas include optimization, distributed control, and stochastic analysis of communication networks, with applications to the Internet, wireless networks, broadband access networks, and content distribution.

His awards include Presidential Early Career Award for Scientists and Engineers 2008 from the White House, Young Investigator Award 2007 from ONR, TR35 Young Innovator Award 2007 from Technology Review, Young Researcher Award Runner-up 2004-2007 from Mathematical Programming Society, CAREER Award 2005 from NSF, as well as Frontiers of Engineering Symposium participant 2008 from NAE and SEAS Teaching Commendation 2007 from Princeton University. He was a Princeton University Howard B. Wentz Junior Faculty and a Hertz Foundation Fellow. His paper awards include ISI citation Fast Breaking Paper in Computer Science, IEEE INFOCOM Best Paper Finalist, and IEEE GLOBECOM Best Student Paper. His guest and associate editorial services include IEEE/ACM Trans. Netw., IEEE Trans. Inform. Theory, IEEE J. Sel. Area Comm., IEEE Trans. Comm., IEEE Trans. Wireless Comm., and J. Optimization and Engineering, and he co-chaired 38th Conference on Information Sciences and Systems.
Hazer Inaltekin (S'04, M'06) received his B.S. degree with High Honors from the Department of Electrical and Electronic Engineering at Bogazici University, Istanbul, Turkey, in 2001. After completing his undergraduate studies in Turkey, he started his M.S./Ph.D. program in the School of Electrical and Computer Engineering at Cornell University. He earned his M.S. and Ph.D. degrees both from Cornell University in April 2005 and in August 2006, respectively.

He was then with the Wireless Intelligent Systems Laboratory at Cornell University as a postdoctoral research scientist until August 2007. He is currently with the Department of Electrical Engineering at Princeton University as a postdoctoral research scientist. His research interests include wireless communications, wireless networks, game theory, information theory, and financial mathematics.

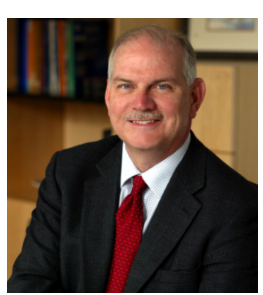

H. Vincent Poor (S'72, M'77, SM'82, F'87) received the Ph.D. degree in EECS from Princeton University in 1977. From 1977 until 1990, he was on the faculty of the University of Illinois at UrbanaChampaign. Since 1990 he has been on the faculty at Princeton, where he is the Michael Henry Strater University Professor of Electrical Engineering and Dean of the School of Engineering and Applied Science. Dr. Poor's research interests are in the areas of stochastic analysis, statistical signal processing and their applications in wireless networks and related fields. Among his publications in these areas are the recent books MIMO Wireless Communications (Cambridge University Press, 2007), coauthored with Ezio Biglieri, et al., and Quickest Detection (Cambridge University Press, 2009), coauthored with Olympia Hadjiliadis.

Dr. Poor is a member of the National Academy of Engineering, a Fellow of the American Academy of Arts and Sciences, and a former Guggenheim Fellow. He is also a Fellow of the Institute of Mathematical Statistics, the Optical Society of America, and other organizations. In 1990, he served as President of the IEEE Information Theory Society, and in 2004-07 he served as the Editor-in-Chief of the IEEE Transactions on Information Theory. $\mathrm{He}$ was the recipient of the 2005 IEEE Education Medal. Recent recognition of his work includes the 2007 IEEE Marconi Prize Paper Award, the 2007 Technical Achievement Award of the IEEE Signal Processing Society, and the 2008 Aaron D. Wyner Award of the IEEE Information Theory Society.

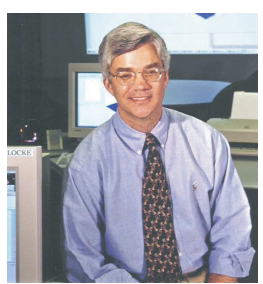

Stephen B. Wicker Stephen B. Wicker (SM'92) received the B.S.E.E. with High Honors from the University of Virginia in 1982. He received the M.S.E.E. from Purdue University in 1983 and a $\mathrm{Ph} . \mathrm{D}$. in Electrical Engineering from the University of Southern California in 1987.

$\mathrm{He}$ is a Professor of Electrical and Computer Engineering at Cornell University, and a member of the graduate fields of Computer Science and Applied Mathematics. Professor Wicker was awarded the 1988 Cornell College of Engineering Michael Tien Teaching Award and the 2000 Cornell School of Electrical and Computer Engineering Teaching Award.

Professor Wicker is the author of Codes, Graphs, and Iterative Decoding (Kluwer, 2002), Turbo Coding (Kluwer, 1999), Error Control Systems for Digital Communication and Storage (Prentice Hall, 1995) and Reed-Solomon Codes and Their Applications (IEEE Press, 1994). He has served as Associate Editor for Coding Theory and Techniques for the IEEE Transactions on Communications, and is currently Associate Editor for the ACM Transactions on Sensor Networks. He has served two terms as a member of the Board of Governors of the IEEE Information Theory Society. 